\title{
Role of P-Glycoprotein Inhibitors in the Bioavailability Enhancement of Solid Dispersion of Darunavir
}

\author{
Saleha Rehman, ${ }^{1}$ Bushra Nabi, ${ }^{1}$ Mohammad Fazil, ${ }^{1}$ Saba Khan, ${ }^{1}$ Naimat Kalim Bari, ${ }^{2}$ \\ Romi Singh, ${ }^{3}$ Shavej Ahmad, ${ }^{3}$ Varinder Kumar, ${ }^{3}$ Sanjula Baboota, ${ }^{1}$ and Javed Ali ${ }^{1}$ \\ ${ }^{1}$ Department of Pharmaceutics, School of Pharmaceutical Education \& Research, Jamia Hamdard, New Delhi 110062, India \\ ${ }^{2}$ Institute of Nanoscience and Technology, Phase X, Mohali, Chandigarh, Punjab, India \\ ${ }^{3}$ Research and Development Centre, Sun Pharmaceuticals Industries Ltd., Gurgaon, Haryana, India \\ Correspondence should be addressed to Javed Ali; javedaali@yahoo.com
}

Received 7 July 2017; Revised 15 September 2017; Accepted 2 October 2017; Published 31 October 2017

Academic Editor: Kamla Pathak

Copyright @ 2017 Saleha Rehman et al. This is an open access article distributed under the Creative Commons Attribution License, which permits unrestricted use, distribution, and reproduction in any medium, provided the original work is properly cited.

\begin{abstract}
Objective. The aim of the present study was to improve bioavailability of an important antiretroviral drug, Darunavir (DRV), which has low water solubility and poor intestinal absorption through solid dispersion (SD) approach incorporating polymer with Pglycoprotein inhibitory potential. Methods. A statistical approach where design of experiment (DoE) was used to prepare SD of DRV with incorporation of P-glycoprotein inhibitors. Using DoE, different methods of preparation, like melt, solvent evaporation, and spray drying method, utilizing carriers like Kolliphor TPGS and Soluplus were evaluated. The optimized SD was characterized by DSC, FTIR, XRD, and SEM and further evaluated for enhancement in absorption using everted gut sac model, effect of food on absorption of DRV, and in vivo prospect. Results and Discussion. DSC, FTIR, XRD, and SEM confirmed the amorphicity of drug in SD. Oral bioavailability studies revealed better absorption of DRV when given with food. Absorption studies and in vivo study findings demonstrated great potential of Kolliphor TPGS as P-glycoprotein inhibitor for increasing intestinal absorption and thus bioavailability of DRV. Conclusion. It is concluded that SD of DRV with the incorporation of Kolliphor TPGS was potential and promising approach in increasing bioavailability of DRV as well as minimizing its extrusion via P-glycoprotein efflux transporters.
\end{abstract}

\section{Introduction}

Acquired Immunodeficiency Syndrome (AIDS) has been one of the most devastating pandemic diseases over the last few decades caused by its etiologic agent Human Immunodeficiency Virus (HIV). Latest reports reveal that globally 40 million people are infected with HIV including 2.1 million from India in 2013 [1]. HIV is responsible for killing helper T-lymphocytes (CD4 ${ }^{+} \mathrm{T}$-cells) which play a key role in the process of gaining immunity to specific pathogens, including HIV itself. No complete cure is possible for people with AIDS and life-long treatment with a combination of antiretroviral drugs; that is, Highly Active Antiretroviral Therapy (HAART) is the only therapeutic intervention with proven efficacy against HIV infection [2,3].

HIV protease inhibitors (PIs) currently are the key components of first-line therapy in both treatment-resistant and treatment-experienced patients. The introduction of novel second-generation PIs such as Darunavir Ethanolate (DRV) with activity against wild type HIV-1 virus and multidrug resistant strains requires at least four concomitant mutations in the viral genome for resistance development, thus providing clinicians with superior drugs to counter the development of resistance [4]. DRV is coadministered with food and low dose Ritonavir (RTV), a potent CYP3A4 inhibitor as a pharmacokinetic booster to result in clinically relevant increase in the systemic exposure (bioavailability increase by up to $40 \%$ ) of DRV $[5,6]$. However, DRV suffer from disadvantages such as low solubility in water $(0.15 \mathrm{mg} / \mathrm{ml})$ and poor intestinal uptake due to drug efflux through active efflux transporter Pglycoprotein (P-gp) and by drug metabolism via Cytochrome P450 (CYP) 3A [7, 8].

The current clinical antiretroviral therapies have suboptimal therapeutic effect attributed to poor bioavailability of anti-HIV drugs which is due to either their poor solubility, extreme first pass metabolism, extrusion into intestine lumen 
by efflux transporters, drug metabolization by enzymes, or poor permeability. Therefore, there is a need for a delivery system to overcome such solubility and bioavailability issues [9]. Solid Dispersion (SD) is considered as one of the most promising strategies to enhance the dissolution profile of poorly aqueous soluble drugs. In the present study, for bioavailability improvement of DRV, two thrust areas were emphasized, firstly increasing its solubility by means of SD and secondly inhibiting its P-gp mediated efflux by incorporating polymer with pharmacokinetic modulatory property [10]. Therefore, SD was opted as a suitable approach for enhancing solubilization of DRV. This formulation strategy of SD combats major concerns related to the other methodologies such as physical stability of the drug, since in many cases the amorphous form readily recrystallizes into the more stable crystalline form losing the advantage of increased dissolution rate and increased solubility and finally compromising the bioavailability of such drugs [11].

Second approach to increase the systemic availability of DRV is to hamper the drug efflux through P-gp. Coadministration of P-gp inhibitors (therapeutic agents) would result in increase in bioavailability but the toxicity associated with their high dose (required for P-gp inhibition) limits their usage. Ritonavir is the most widely used therapeutic agent for the inhibition of P-gp efflux pumps, thus contributing as a pharmacokinetic booster when given with antiretroviral therapies [12]. Pharmaceutical excipients, which are largely used as inert vehicles in drug formulations with no pharmacological actions of its own, are emerging as a special class of P-gp inhibitors [13]. Pharmaceutical surfactants which have already been approved for use in pharmaceutical formulations seem to be a better choice since they interact directly with the lipid bilayer plasma membrane, inserting themselves in between them and thereafter fluidizing them. Some of these surfactants include C8/C10 Glycerol and PEG Esters, Sucrose Esters, Polysorbates, and Tocopherol Esters [12].

Simple carriers without surfactant properties have been used earlier in order to enhance bioavailability but the carriers with surfactant properties have not been investigated to a wide extent as they possess potential to achieve anticipated bioavailability. These carriers with surfactant like properties have given manifold improved bioavailability in comparison to simple carriers and hence can be preferred over them. Interestingly, utilizing the carriers which possess P-glycoprotein inhibitory activity could potentially enhance the bioavailability of substrate drugs and thus will further add to the therapeutic effect. Therefore, in the present work, Kolliphor as the carrier which exhibits surfactant and Pgp inhibitory activity has been used in order to enhance solubility resulting in enhancement of related bioavailability of an anti-HIV drug, Darunavir (DRV). However, there is no previous study reporting the preparation of solid dispersion of DRV. Some formulation approaches attempted in the past aiming to increase the bioavailability of DRV were complexation of DRV to $\beta$-cyclodextrin [14] and production of pellets of DRV using wet extrusion/spheronization with kappacarrageenan or microcrystalline cellulose (MCC) as pelletization aid [15]. However, both the approaches were targeted to increase the solubility and bioavailability of DRV but did not focus on increasing the intestinal uptake by inhibition of P-gp transporters present on the intestinal epithelium.

In the present study, SDs of DRV were prepared by employing design of experiment (DoE) to investigate different methods of preparation (like melt, solvent evaporation, and spray drying) and to screen and optimize carriers which are surface-active agents and P-gp inhibitors. The effect of food on absorption of drug was also studied both in vitro (in biorelevant media) and in vivo. Therefore, the formulation prepared by using this strategy is hypothesized to increase the solubility of the poorly soluble drug DRV as well as inhibiting its efflux by P-gp which can lead to an improved in vivo prospect and increased therapeutic efficacy. To our knowledge, this is the first study reporting the formulation of SD of DRV using novel polymer Kolliphor TPGS exhibiting P-gp inhibiting potential.

Till date, third-generation carrier has not been used for the bioavailability enhancement of an anti-HIV agent. Furthermore, Darunavir is an anti-HIV agent which has not been extensively worked upon. Only few research papers are available whose rationale was entirely different from the rationale of the present work. Although the concept of using P-gp inhibitor has also been investigated by a large number of research groups, the use of third-generation carrier with Pgp inhibiting activity for the solubility and bioavailability enhancement of an anti-HIV agent has not been studied previously. Therefore, the present work is novel and remains critically unexplored so far.

\section{Material and Method}

2.1. Material. Darunavir Ethanolate, Ritonavir, and Atazanavir Sulphate were received as a gift sample from Ranbaxy Research Laboratory (Gurgaon, India). Soluplus, Kolliphor TPGS (d-alpha-tocopheryl polyethylene glycol 1000 succinate), and Poloxamer 188 were obtained from BASF (Ludwigshafen, Germany). PVP K30 (Polyvinylpyrrolidone K30) was obtained from ISP (New Jersey, USA) and HPMC E5 (hydroxypropyl methylcellulose) from Dow Chemical Company (Michigan, USA). Diethyl ether and ethanol were obtained from Merck (Mumbai, India). SIF (Simulated Intestinal Fluid) powder was from Phares AG (Basel, Switzerland). Other chemicals and reagents used were from SD Fine Chemicals, Ltd. (Mumbai, India), and Qualigens Fine Chemical (Mumbai, India) and were of analytical grade. All drug solutions and buffer solutions were freshly prepared before use.

\subsection{Formulation Design and Development}

2.2.1. Selection of Carrier. From literature search, six different carriers possessing P-gp inhibiting activity like Poloxamer 188, Kolliphor TPGS, Tween 80, Soluplus, Povidone (PVP K30), and Hypromellose (HPMC E5) were selected and were screened out by phase solubility study to determine the most appropriate and suitable carrier. In phase solubility study, an excess amount of DRV (100 mg) was added to $10 \mathrm{~mL}$ of distilled water, each containing different concentrations of carriers (i.e., $0.5 \%, 1 \%, 1.5 \%$, and $2 \% \mathrm{w} / \mathrm{v}$ ). The samples were 
TABLE 1: Saturation solubility and \% drug content of eighteen batches of solid dispersion (as predicted by Simple Custom Design) using Soluplus and Kolliphor TPGS. Data presented as mean \pm SD, $n=3$ for each formulation $(n=3)$.

\begin{tabular}{|c|c|c|c|c|c|c|}
\hline Exp. & Formulation code & Polymer & Polymer : drug & Method & $\begin{array}{l}\text { Saturation solubility } \\
(\mathrm{mg} / \mathrm{ml}) \text { mean } \pm \mathrm{SD}\end{array}$ & $\%$ drug content \\
\hline 1 & SD1 & $\mathrm{P} 1$ & $0.5: 1$ & SE & $0.38 \pm 0.005$ & $98.16 \pm 0.906$ \\
\hline 2 & SD2 & P1 & $2: 1$ & S.D & $1.12 \pm 0.012$ & $96.95 \pm 0.527$ \\
\hline 3 & SD3 & $\mathrm{P} 2$ & $0.5: 1$ & MM & $0.59 \pm 0.009$ & $88.58 \pm 1.238$ \\
\hline 4 & $\mathrm{SD} 4$ & $\mathrm{P} 2$ & $0.5: 1$ & $\mathrm{SE}$ & $0.54 \pm 0.012$ & $91.65 \pm 0.951$ \\
\hline 5 & SD5 & $\mathrm{P} 1$ & $0.5: 1$ & S.D & $0.59 \pm 0.006$ & $91.81 \pm 0.638$ \\
\hline 6 & SD6 & $\mathrm{P} 2$ & $1.25: 1$ & SE & $0.84 \pm 0.018$ & $93.23 \pm 0.422$ \\
\hline 7 & SD7 & $\mathrm{P} 2$ & $2: 1$ & S.D & $1.36 \pm 0.025$ & $99.93 \pm 0.974$ \\
\hline 8 & SD8 & $\mathrm{P} 1$ & $2: 1$ & SE & $0.98 \pm 0.013$ & $89.42 \pm 0.894$ \\
\hline 9 & SD9 & $\mathrm{P} 1$ & $2: 1$ & MM & $0.69 \pm 0.044$ & $74.07 \pm 0.637$ \\
\hline 10 & SD10 & $\mathrm{P} 2$ & $2: 1$ & SE & $1.02 \pm 0.014$ & $93.69 \pm 0.691$ \\
\hline 11 & SD11 & $\mathrm{P} 2$ & $1.25: 1$ & MM & $0.74 \pm 0.007$ & $90.44 \pm 0.721$ \\
\hline 12 & SD12 & $\mathrm{P} 1$ & $1.25: 1$ & SE & $0.42 \pm 0.014$ & $88.39 \pm 0.418$ \\
\hline 13 & SD13 & $\mathrm{P} 2$ & $1.25: 1$ & S.D & $1.18 \pm 0.015$ & $99.09 \pm 0.536$ \\
\hline 14 & SD14 & $\mathrm{P} 1$ & $1.25: 1$ & MM & $0.59 \pm 0.041$ & $67.09 \pm 0.851$ \\
\hline 15 & SD15 & $\mathrm{P} 2$ & $0.5: 1$ & S.D & $0.83 \pm 0.009$ & $97.14 \pm 0.711$ \\
\hline 16 & SD16 & $\mathrm{P} 2$ & $2: 1$ & MM & $0.99 \pm 0.024$ & $92.21 \pm 0.632$ \\
\hline 17 & SD17 & $\mathrm{P} 1$ & $1.25: 1$ & S.D & $0.95 \pm 0.012$ & $93.51 \pm 0.847$ \\
\hline 18 & SD18 & $\mathrm{P} 1$ & $0.5: 1$ & MM & $0.36 \pm 0.048$ & $59.19 \pm 0.939$ \\
\hline
\end{tabular}

$\mathrm{P} 1=$ Soluplus, $\mathrm{P} 2$ = Kolliphor TPGS, MM = melt method, $\mathrm{SE}=$ solvent evaporation, and SD = spray drying.

prepared in triplicate and shaken in an oscillating water bath (Metrex Scientific Instrument, New Delhi, India) thermostatically controlled at $37 \pm 0.5^{\circ} \mathrm{C}$ for $72 \mathrm{~h}$ and then centrifuged (Remi R-244, Mumbai, India) at 4,000 rpm for $15 \mathrm{~min}$. The samples were filtered through a $0.45 \mu \mathrm{m}$ membrane filter, suitably diluted, and analyzed spectrophotometrically by UV spectrophotometer UV -2450 (Shimadzu, Kyoto, Japan) at $264 \mathrm{~nm}[16]$.

2.2.2. Custom Design. Soluplus and Kolliphor TPGS were selected based on the phase solubility study as a carrier. From the design of experiment (DoE), custom design was applied to screen (i) method of preparation of solid dispersion, (ii) polymer as a carrier, and (iii) drug to polymer ratio as input variables. The custom design studied the interaction of various categorical and continuous parameters and their effect on the dissolution rate of the drug and on cumulative percentage drug release. The polymer (Soluplus and Kolliphor TPGS) and the method of preparation (melt, solvent evaporation, and spray drying) were selected as the categorical factor and the drug:polymer ratio (1:0.5, 1:1.25, and $1: 2)$ was selected as the continuous factor. The response factor (output variable) of the study was \% cumulative drug release at $30 \mathrm{~min}$ and $60 \mathrm{~min}$. A set of 18 experiments were generated by software (JMP software version 9) (Table 1). Experiments were performed in same randomization sequence as given by software.

\subsubsection{Preparation of Solid Dispersion}

Solid Dispersion by Melt Method. Accurately weighed quantity of polymer (Soluplus or Kolliphor) as per experiment numbers $3,9,11,14,16$, and 18 of DoE was taken in a china dish and melted over a hot plate (Scientific Equipment, New Delhi, India) at temperature above melting point of polymer (melting point of Soluplus $90 \pm 2^{\circ} \mathrm{C}$ and of Kolliphor TPGS $60 \pm 2^{\circ} \mathrm{C}$ ). The drug was weighed and added to the molten mass of polymer and the mixture was stirred continuously for 10 min using a glass rod and then allowed to cool in an ice bath. The dispersion of drug prepared using Soluplus was solidified, scraped, pulverized, sieved with BSS (British Standard Sieve) sizes, sieve number 40, and stored in a desiccator. The dispersions obtained using Kolliphor TPGS were semisolid waxy substance which were scraped and stored in a desiccator.

Solid Dispersion by Solvent Evaporation Method. Accurately weighed quantity of polymer (Soluplus or Kolliphor) as per experiment numbers $1,4,6,8,10$, and 12 of DoE was dissolved in ethanol in a beaker using an overhead stirrer (RQ 124A, Universal Motors Ltd., Mumbai, India). Accurately weighed amount of DRV was then added to the polymer solution with continuous stirring for around 45-60 min until a clear solution was obtained. The clear solution was poured in Petriplates and dried at $40 \pm 2{ }^{\circ} \mathrm{C}$ in an oven under vacuum. The dried samples were scraped, pulverized, sieved with BSS sieve number 40 , and kept in a desiccator.

Solid Dispersion by Spray Drying Method. As per experiment numbers 2, 5, 7, 13, 15, and 17 of DoE, accurate quantity of Soluplus was dissolved in ethanol and Kolliphor TPGS was dissolved in dichloromethane (DCM) using an overhead stirrer. Accurately weighed amount of DRV was then added 
to the respective polymer solutions with continuous stirring for around 45-60 min until a clear solution was obtained. This final clear solution was used as a feeding solution and sprayed via a fluid nozzle from a Mini Spray Dryer (Buchi, Switzerland). Nitrogen gas was used as the drying gas at an atomization pressure of 4 bars with the aspirator set to $100 \%$ so that the oxygen concentration remains below $6 \%$. The flow rate of the feed solution was set to $5 \mathrm{ml} / \mathrm{min}$. After the completion of the spray drying step, the samples were transferred into a freeze dryer and freeze dried for $72 \mathrm{~h}$. The powder finally obtained was further dried in a vacuum dryer for $24 \mathrm{~h}$ and then sieved through sieve BSS 40.

\subsection{Evaluation of Solid Dispersion}

2.3.1. Saturation Solubility Study. Saturation solubility study was carried out to determine the increase in the solubility of pure drug in the prepared SDs. Excess amount of the prepared SDs was added to $10 \mathrm{ml}$ of distilled water in glass vials. Samples were kept in triplicate on a water bath shaker at $37 \pm 0.5^{\circ} \mathrm{C}$ for $48 \mathrm{~h}$, after which they were filtered through $0.45 \mu \mathrm{m}$ PVDF (polyvinylidene fluoride) membrane filters (from Millipore), suitably diluted, and analyzed by UV spectrophotometer at $264 \mathrm{~nm}$.

2.3.2. Drug Content Determination. SDs equivalent to $80 \mathrm{mg}$ of DRV were added to $250 \mathrm{ml}$ of methanol contained in a volumetric flask. Samples were sonicated for $15 \mathrm{~min}$ and filtered using a $0.45 \mu \mathrm{m}$ PVDF filter. After suitable dilution, the drug content was determined using the calibration curve of pure drug in methanol by UV analysis.

2.3.3. In Vitro Dissolution Study. Dissolution studies were performed using a USP II Paddle dissolution apparatus (Distek, USA). The prepared SDs were filled in 00 size capsules and placed in Japanese sinkers. The sinkers were then placed in dissolution vessels containing $900 \mathrm{ml}$ of acetate buffer $\mathrm{pH}$ 4.5 , at $75 \mathrm{rpm}$ and temperature $37 \pm 0.5^{\circ} \mathrm{C}$. Samples $(5 \mathrm{ml})$ were collected periodically at $15,30,45,60$, and $90 \mathrm{~min}$, filtered through a $0.45 \mu \mathrm{m}$ PVDF filter and replaced with a fresh dissolution medium $(5 \mathrm{ml})$. The concentrations were analyzed at $264 \mathrm{~nm}$ by UV spectrophotometer.

2.4. Physicochemical Characterization of Optimized Solid Dispersion. Differential Scanning Calorimetry (DSC) was performed for DRV, Kolliphor TPGS, and SD7 in Jade DSC (Perkin Elmer, Massachusetts, USA). The samples were heated in a temperature range of $30-300^{\circ} \mathrm{C}$ in pierced aluminium pans. The heating rate was set to $10^{\circ} \mathrm{C} / \mathrm{min}$. Inert atmosphere was maintained by purging nitrogen gas at a flow rate of $20 \mathrm{ml} / \mathrm{min}$.

Fourier Transform Infrared (FTIR) analysis was carried out with the help of Bruker alpha-T spectrophotometer, Ettlingen, Germany. Samples equivalent to $5 \mathrm{mg}$ were placed on the sample holder. The overhead compressor was put on the sampling point to allow transmittance to occur. The spectra were recorded by scanning the pellet between 4000 and 400 wavelengths $\left(\mathrm{cm}^{-1}\right)$.
X-Ray Diffraction (XRD) analyses were performed using an X-ray diffractometer Bruker D8 ADVANCE (Bruker, USA), equipped with CU-anode. Copper was the source of radiation operated at $30 \mathrm{kV}, 40 \mathrm{~mA}$, and a nickel filter was used to strip $\mathrm{K}$ beta radiation. The shift or change in the $2 \theta$ values was obtained.

Scanning Electron Microscopy (SEM) helped in determining surface morphology of DRV and its SD. Prior to examination, samples were mounted on an aluminium stub using a double sided adhesive tape and then making it electrically conductive by coating with a thin layer of platinum (approximately $20 \mathrm{~nm}$ ) in vacuum. It was operated at an acceleration voltage of $1.9 \mathrm{kV}$.

2.5. Dissolution Studies in FaSSIF and FeSSIF Media. The dissolution profile of the SD7 was also performed in FaSSIF (fasted state simulated intestinal fluid) and FeSSIF (fed state simulated intestinal fluid) media to determine the effect of food on the absorption of DRV in the intestinal tract. These two biorelevant media, FaSSIF and FeSSIF, were developed to simulate the condition of the intestine in the fasted and fed states [17].

2.5.1. Preparation of FaSSIF Media. Blank FaSSIF was prepared by dissolving $6.186 \mathrm{~g} \mathrm{NaCl}, 4.47 \mathrm{~g} \mathrm{NaH} \mathrm{NO}_{4}$, and $0.348 \mathrm{~g} \mathrm{NaOH}$ in $900 \mathrm{ml}$ of distilled water and the $\mathrm{pH}$ was adjusted to 6.5 by using $1 \mathrm{~N} \mathrm{NaOH}$ or $\mathrm{HCl}$ using $\mathrm{pH}$ meter. The volume was made up to $1000 \mathrm{ml}$ using distilled water.

Blank FaSSIF medium $(500 \mathrm{ml})$ was taken and $2.240 \mathrm{~g} \mathrm{SIF}$ powder was added to it and this solution was magnetically stirred until the powder completely dissolved to obtain a clear micellar solution. The volume was made up to $1000 \mathrm{ml}$ using the buffer. The solution was allowed to stand for $2 \mathrm{~h}$; it becomes slightly opalescent and thereafter used. A volume of $500 \mathrm{ml}$ is recommended for dissolution for simulating fasted state condition

2.5.2. Preparation of FeSSIF Media. Blank FeSSIF was prepared by dissolving $8.65 \mathrm{~g}$ glacial acetic acid, $11.874 \mathrm{~g} \mathrm{NaCl}$, and $4.04 \mathrm{~g} \mathrm{NaOH}$ in $900 \mathrm{ml}$ of distilled water and the $\mathrm{pH}$ was adjusted to 5.0 by using $1 \mathrm{~N} \mathrm{NaOH}$ or $\mathrm{HCl}$ using $\mathrm{pH}$ meter. The volume was made up to $1000 \mathrm{ml}$ using distilled water.

Blank FeSSIF medium $(500 \mathrm{ml})$ was taken and $11.20 \mathrm{~g} \mathrm{SIF}$ powder was added to it and this solution was magnetically stirred until the powder completely dissolved to obtain a clear micellar solution. The volume was made up to $1000 \mathrm{ml}$ using the buffer and thereafter used. For simulating fed state condition $1 \mathrm{~L}$ dissolution fluid is recommended.

2.6. In Vitro Permeation Study of the Optimized Solid Dispersion. In vitro permeation study of the optimized SD (SD7) was carried out with the help of everted gut sac method. The present study was aimed at investigating the effect of Kolliphor TPGS as P-gp inhibitor on the intestinal absorption of DRV. The experiments were performed on adult male albino Wistar rats (150-200 g) obtained from Central Animal House, Jamia Hamdard (Approval number 1075). They were housed in cages with a $12 \mathrm{~h}$ light/dark cycle with free access 
to water and maintained on the feed. After overnight fasting $(10-12 \mathrm{~h})$, the rats were anesthetized with diethyl ether and the small intestine at the ileocecal junction was isolated and rinsed with Tyrode buffer solution $\mathrm{pH} 7.4$ (containing in $\mathrm{mM}$ : 15 glucose, $11.90 \mathrm{NaHCO}_{3}, 136.9 \mathrm{NaCl}, 4.2 \mathrm{NaH}_{2} \mathrm{PO}_{4}, 2.7 \mathrm{KCl}$, $1.2 \mathrm{CaCl}_{2}$, and $0.5 \mathrm{MgCl}_{2}$ ) at room temperature. The intestinal segment was immediately transferred to oxygenated Tyrode solution $\left(95 \% \mathrm{O}_{2}\right.$ and $\left.5 \% \mathrm{CO}_{2}\right)$ maintained at $37 \pm 0.5^{\circ} \mathrm{C}$ and was cleared off the adhering tissue and rinsed with Tyrode solution. The tissue was continuously aerated (oxygenated) with the aid of an electrical aerator. Intestinal segments of $4 \mathrm{~cm}$ were cut and ligated with nylon thread at one end and carefully everted on the glass rod. The everted gut sacs were filled with $500 \mu \mathrm{l}$ of Tyrode solution and ligated at the other end and then placed inside the test tube containing $15 \mathrm{~mL}$ of the test solution continuously bubbled with atmospheric air at 15-20 bubbles per min separately. Test tubes were maintained at temperature $37 \pm 0.5^{\circ} \mathrm{C}$ on water bath. The test solution outside the sac was termed as mucosal fluid, and the solution inside the sac was termed as serosal fluid. The amount of drug permeated across the intestine in serosal fluid was determined using HPLC method after predetermined time intervals $(15,30,45,60,90$, and $120 \mathrm{~min})$; HPLC with UV detector method was employed for quantification of DRV in SD formulation. The mobile phase used for quantification of DRV consisted of acetonitrile and water $(40: 60)$ at flow rate of $1 \mathrm{ml} / \mathrm{min}$. The retention time $\left(R_{t}\right)$ was $4.192 \mathrm{~min}$ [18].

The experiment was conducted on three formulations including pure drug DRV ( $15 \mathrm{ml}$ of $1 \mathrm{mg} / \mathrm{ml}$ solution), the conventional treatment, that is, DRV + Ritonavir (RTV) $(15 \mathrm{ml}$ of $1 \mathrm{mg} / \mathrm{ml} \mathrm{DRV}+1.7 \mathrm{ml}$ of $1 \mathrm{mg} / \mathrm{ml} \mathrm{RTV}$ calculated according to the dose of $8 \mathrm{mg} / \mathrm{kg}$ ) and SD7 (15 ml equivalent to $1 \mathrm{mg} / \mathrm{ml}$ of DRV). Permeability coefficients $\left(P_{\text {app }}\right)$ of these formulations were calculated from mucosal to serosal direction according to the equation:

$$
P_{\text {app }}(\mathrm{cm} / \mathrm{sec})=\frac{(d Q / d t)}{\left(A * C_{o}\right)},
$$

where the $d Q / d t$ is the rate of drug permeation across the tissue, $A$ is the cross-sectional area of the tissue, and $C_{o}$ is the initial concentration in the donor compartment at $t=0$ [19].

2.7. Pharmacokinetic Studies. The pharmacokinetic studies were performed to compare the plasma concentration profiles of the optimized SD with the pure drug and conventional therapy (DRV + RTV) to check the bioavailability difference between the two. The animal species used for in vivo experiments were adult albino Wistar male rats weighing 150-200 g obtained from Central Animal House, Jamia Hamdard. The research protocol of the animal experimentation was approved by Institutional Animal Ethics Committee (IAEC) of Hamdard University, New Delhi, India. The animals were housed four per cage at $20-24^{\circ} \mathrm{C}$ and $55 \pm 5 \%$ relative humidity with free access to food and water with a 12-h light-dark cycle.

2.7.1. Estimation of DRV in Plasma. A rapid, simple, specific, and accurate high performance liquid chromatography
(HPLC) with UV detector method was employed for quantification of DRV in SD formulation in blood plasma as reported by Takahashi et al., 2007 [20]. The mobile phase used for quantification of DRV consisted of $39 \% 50 \mathrm{mM}$ phosphate buffer ( $\mathrm{pH} 5.9$ ), 22\% methanol, and 39\% acetonitrile at flow rate of $1 \mathrm{ml} / \mathrm{min}$. The retention time $\left(R_{t}\right)$, detection limit, and quantification limit were $3.458 \mathrm{~min}, 32.14 \mathrm{ng} / \mathrm{ml}$, and $51.87 \mathrm{ng} / \mathrm{ml}$, respectively. Internal standard used was Atazanavir. The accuracy and precision were excellent. The same method was employed for both fed and fasted conditions.

2.7.2. Animals and Dosing Protocol. The albino Wistar rats were divided into two groups: one group contains the rats which were fasted overnight (fasted state), while the other group contains the rats which were fasted overnight and then fed for $15 \mathrm{~min}$ prior to dosing (fed state).

The dose was calculated according to the formula:

$$
\begin{aligned}
& \text { Animal Dose }(\mathrm{mg} / \mathrm{kg}) \\
& =\operatorname{HED}(\mathrm{mg} / \mathrm{kg}) \\
& \quad \times\left(\frac{K_{m} \text { factor for human adult }}{K_{m} \text { factor for rat }}\right),
\end{aligned}
$$

where HED is human equivalent dose in $\mathrm{mg} / \mathrm{kg}, K_{m}$ factor for human adult equals 37, and $K_{m}$ factor for rat equals 6

The fasted and fed groups were further subdivided into four groups with three animals in each group, one for control, the second for pure drug solution of DRV, third for the conventional treatment DRV + RTV, and fourth for the optimized preparation SD7. All the formulations were administered at the required dose $(70 \mathrm{mg} / \mathrm{kg}$ of DRV, $8 \mathrm{mg} / \mathrm{kg}$ of Ritonavir) and were given orally using oral feeding sonde. The blood samples collected at appropriate time intervals were then analysed by HPLC and the pharmacokinetic parameters were estimated.

\section{Results}

3.1. Selection of Carrier. On the basis of the phase solubility study of DRV in different polymers, Soluplus and Kolliphor TPGS were selected as the two carriers for the formulation of SD based on the highest increase in the solubility of the drug in their respective aqueous solutions. The solubility of the drug in pure water was $0.15 \mathrm{mg} / \mathrm{ml}$ and in Soluplus and Kolliphor TPGS solution at drug: polymer ratio of 1:2 was 0.823 and $0.922 \mathrm{mg} / \mathrm{ml}$, respectively. Thus, there was 5-6 times increase in the solubility of drug in water using these carriers (the data is provided as Supplementary Material in Table S.1 available online at https://doi.org/10.1155/2017/8274927).

3.2. Custom Design Analysis. The response variables generated for the runs of the custom design were analyzed to get the optimized formulation. The output variables (response variables) of the study were \% cumulative drug release at 30 and $60 \mathrm{~min}$. Regression plot for different output variables was obtained and the $P$ value for both the regression plots was below 0.05 and $R^{2}$ value was very close to 1 in both 
the cases. Based on this outcome, we concluded that the model is statistically relevant and could predict the response with minimum prediction error (the data is provided as Supplementary Material in Figure S.1).

\subsection{Optimization of Solid Dispersion}

3.3.1. Saturation Solubility Studies. The solubility profile of the prepared SDs was found to be directly proportional to the polymer concentration showing an increase with the increase of polymer concentration from 0.5 parts to 2 parts of the drug (Table 1). SD7 showed the highest saturation solubility of $1.36 \mathrm{mg} / \mathrm{ml}$ increasing the solubility of the drug by up to 9 times.

3.3.2. Drug Content Determination. The results revealed that the drug content was found to be more in the SDs prepared using Kolliphor TPGS than with Soluplus. The highest \% drug content was found to be $99.93 \pm 0.341 \%$ for formulation SD7 (Table 1). The SDs prepared using Soluplus, on the other hand, exhibited a nonuniform pattern for the percent drug content, specifically those obtained by melt method.

3.3.3. In Vitro Dissolution Study. The goal of the dissolution study was to illustrate the improvement in dissolution rate of various SDs over pure drug. Impact of polymer and method of SD preparation on drug release was studied through design of experiment. The output variables (response variables) of the design were $\%$ cumulative drug release at 30 and $60 \mathrm{~min}$. The influence of individual parameters and their interaction terms on the response variables is known as Parameters Estimates.

Parameters for which Prob $>|t|$ value was less than 0.05 had significant impact on response variable. For response of $\%$ drug release at $60 \mathrm{~min}$, all individual terms (main effects) and 2nd-order interaction of polymer:drug ratio with polymer and method were significant terms and had significant impact on response variable (the data is provided as Supplementary Material in Figures S.2 and S.3).

The prediction profiles were generated through the software during the custom design analysis as shown in Figure 1. Prediction profile showed graphical representation of correlation between input variables and output variables.

In Figures 1(a)-1(c), polymer Soluplus was selected and method was changed from solvent evaporation to spray drying to see the impact of different methods on response variables. The $\%$ drug release was found to increase with the increase in polymer: drug ratio. The polymer:drug ratio showed its impact on \% drug release only in case of spray drying method, whereas for the melt and solvent evaporation method, negligible effect of polymer: drug ratio was seen. Thus, out of the three methods, spray drying was found to be the best method for solubility enhancement of drug. The desirability value obtained (0.283) was also the highest when spray drying method was used to make the solid dispersion.

In Figures 1(d)-1(f), Kolliphor TPGS was selected as the polymer and the method was changed to see the impact of different methods on response variables. In comparison to solvent evaporation and hot melt method, spray drying was found to be the best method for solubility enhancement of drug. The desirability value was also found to be highest, that is, 0.639, when choosing spray drying method. Line representing polymer: drug ratio was more inclined when spray drying method was selected for prediction of response variables. The polymer: drug ratio too exhibited its significant effect when spray drying method is selected for the preparation of solid dispersion.

Drug release was found to be the highest $98.8 \%$, for the dispersion prepared by spray drying method using Kolliphor TPGS as the polymer in the drug:polymer ratio of $1: 2$ (SD7) which was almost 3 times of the drug release obtained with the drug alone i.e. $30.9 \%$. However, the maximum drug release obtained by the dispersions using Soluplus as the polymer (SD2) by the same preparation method (spray drying) after $90 \mathrm{~min}$ was comparatively less i.e. $56.3 \%$ (Table 2). Hence the drug release was found to be dependent on the polymer used as well as the method of preparation.

3.4. Characterization of Optimized Solid Dispersion. The optimized formulation, that is, SD7 prepared using Kolliphor TPGS as the polymer in the drug:polymer ratio of $1: 2$ by spray drying method, was characterized by the following methods. DSC thermograms showed the melting endothermic peak for pure DRV at $103.3^{\circ} \mathrm{C}$ [Figure 2(a)] and at $39^{\circ} \mathrm{C}$ for Kolliphor TPGS [Figure 2(b)]. The thermogram of SD7 showed different thermal behaviour for DRV and peak characteristic to the polymer at $37.8^{\circ} \mathrm{C}$ was observed [Figure 2(c)].

The FTIR spectra of SD7 showed disappearance of peaks at 3448 and $1710 \mathrm{~cm}^{-1}$ which are characteristic of DRV. No sharp peak of DRV appeared in this region. However, there is a shift in the peak of polymer in the spectra and peaks at 2868 and $1093 \mathrm{~cm}^{-1}$ characteristic of Kolliphor TPGS are retained in the SD (the data is provided as Supplementary Material in Figure S.4).

Polymorphic transformation was observed with help of $\mathrm{X}$-ray diffraction and results were found to be in good agreement with those of DSC. The crystalline nature of the drug was further confirmed by the appearance of sharp multiple peaks obtained in the XRD spectrum [Figure 3(a)], whereas these peaks were found to disappear in the case of spray dried dispersion confirming decrease in crystallinity [Figure 3(c)]. The observed few intensity peaks in the diffractograms are corresponding to those present in the polymer Kolliphor TPGS [Figure 3(b)].

The SEM images indicated the morphology of the drug to be in crystalline rod shape [Figure 4(a)], whereas the smooth texture in SD depicts its amorphous character [Figure 4(b)]. It was also observed that the dimensions of the drug particles were much smaller in SD7.

3.5. Dissolution Studies in FaSSIF and FeSSIF Media. In vitro dissolution in biorelevant media (FaSSIF and FeSSIF) was performed for DRV and SD7 in order to observe the effect of food on the absorption of drug. The release of drug from SD7 increased from $72.6 \pm 0.439$ in FaSSIF to a maximum percent 


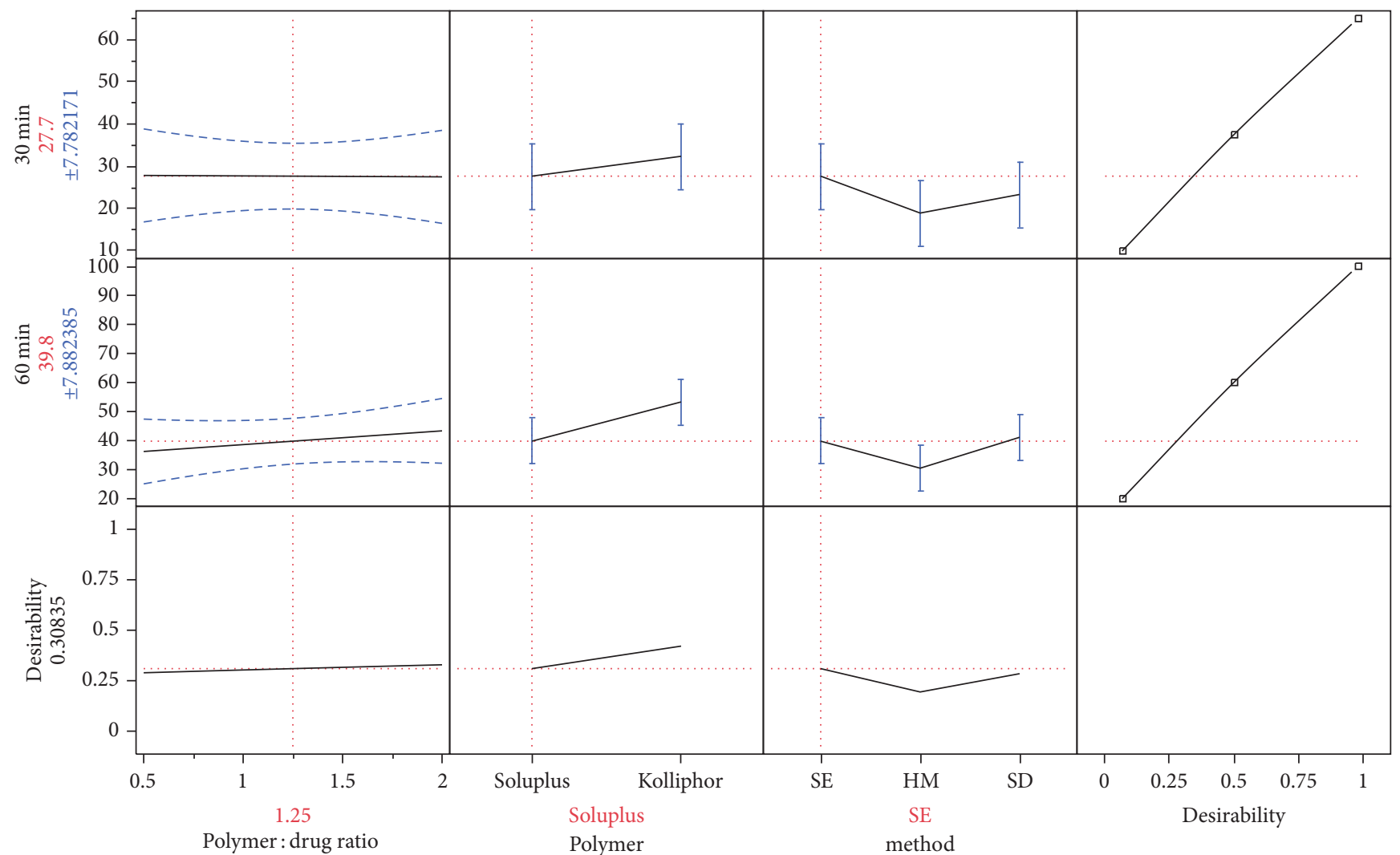

(a)

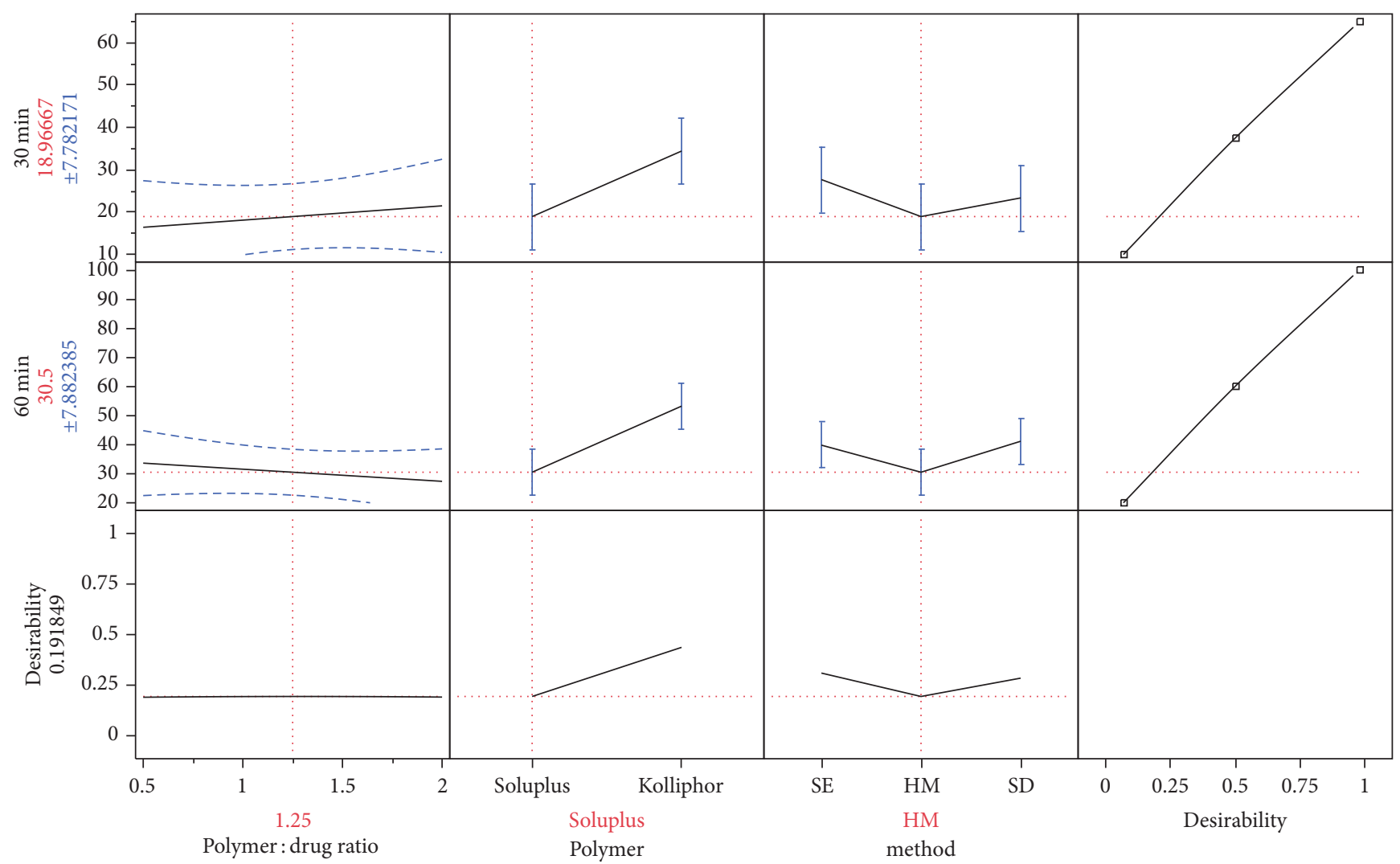

(b)

Figure 1: Continued. 


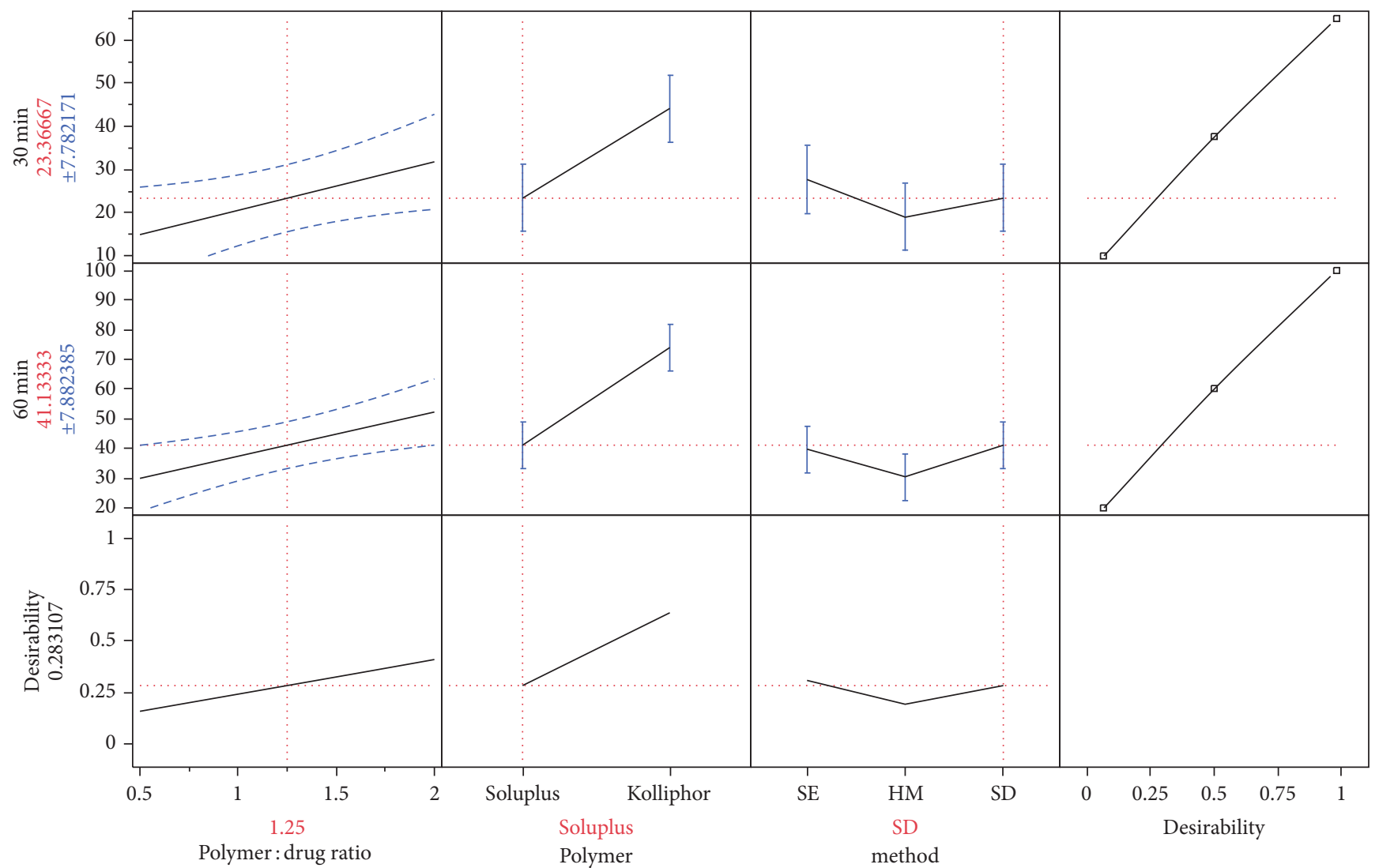

(c)

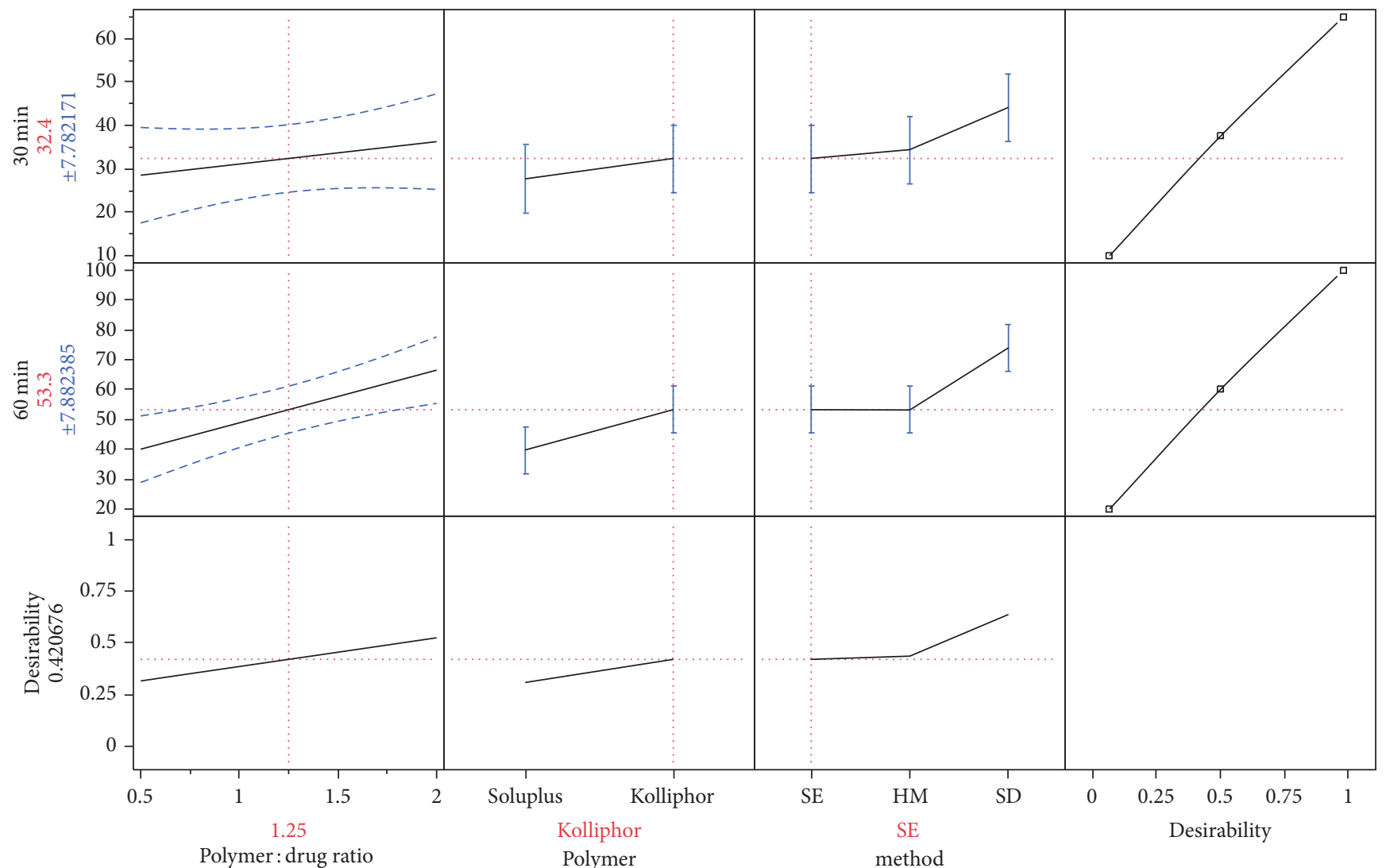

(d)

Figure 1: Continued. 


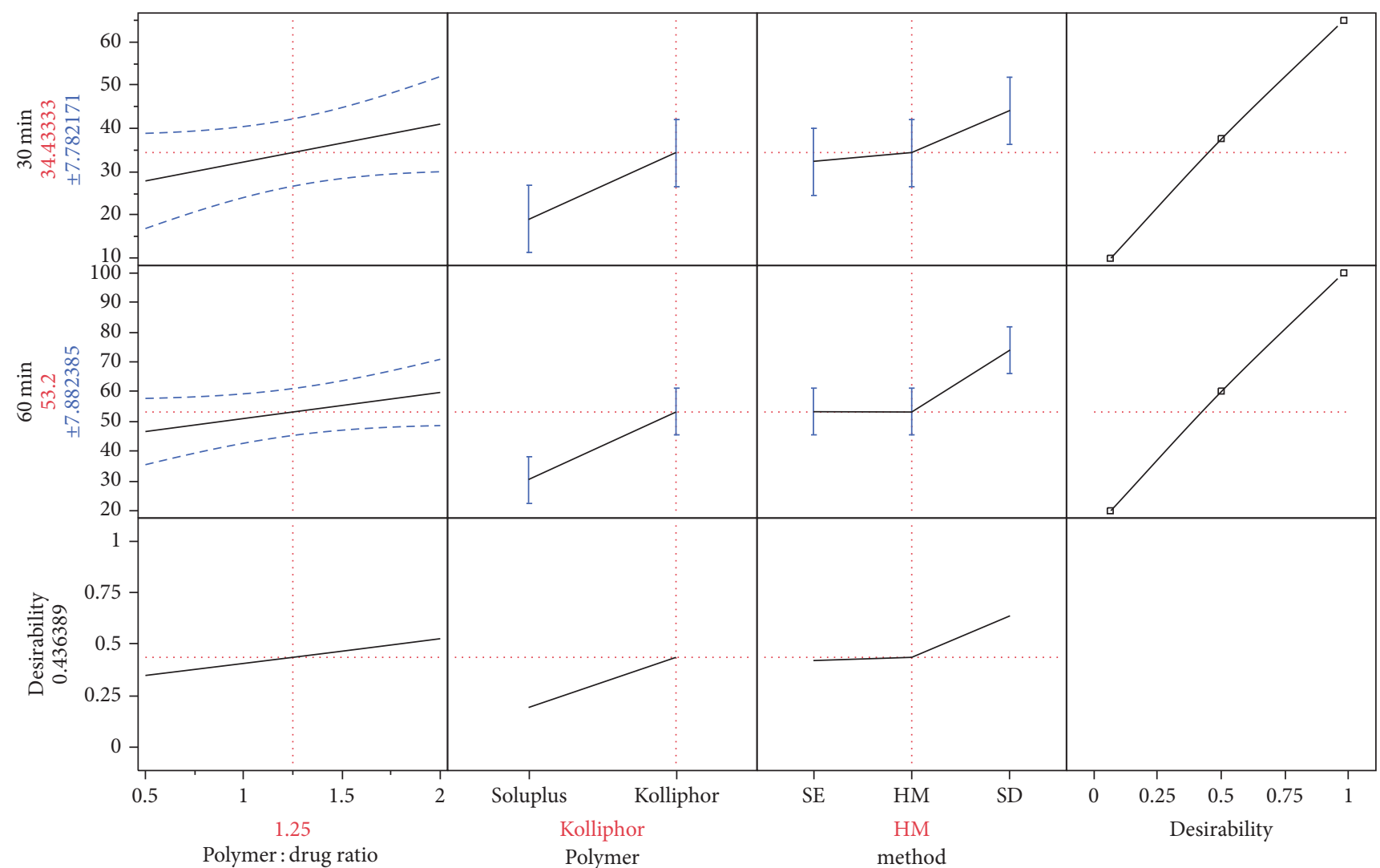

(e)

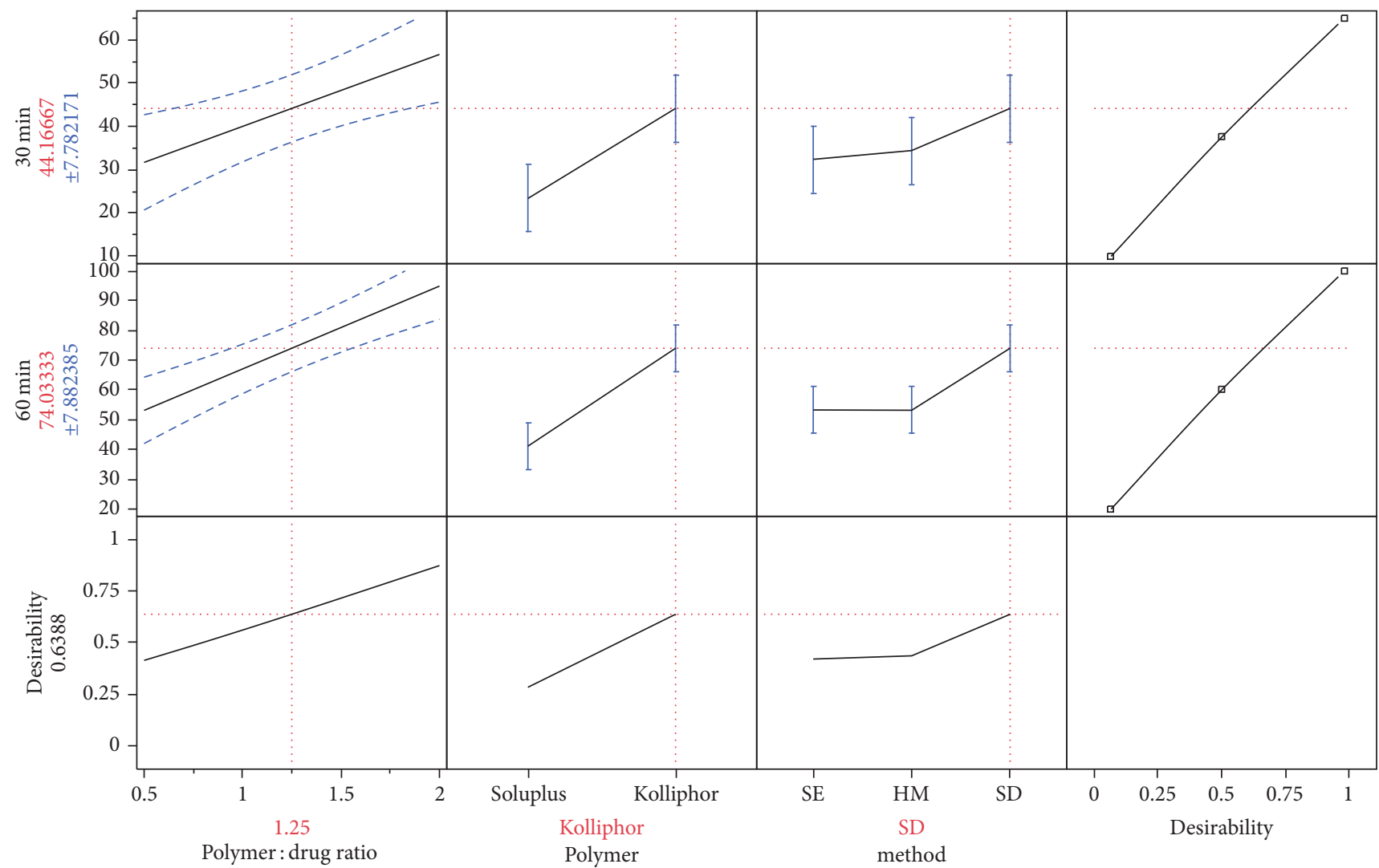

(f)

FIgURE 1: Prediction profiles generated by JMP software (version 9). 
TABLE 2: Comparative dissolution profile of nine batches of SD prepared using polymer P1 (Soluplus) and P2 (Kolliphor TPGS) in acetate buffer $\mathrm{pH}$ 4.5. Data presented as mean $\pm \mathrm{SD}, n=3$ for each formulation.

\begin{tabular}{|c|c|c|c|c|}
\hline \multirow{2}{*}{ Formulation code } & \multirow{2}{*}{ Polymer } & \multicolumn{3}{|c|}{ Cumulative $\%$ drug release $( \pm \mathrm{SD})(n=3)$} \\
\hline & & After $30 \mathrm{~min}$ & After $60 \mathrm{~min}$ & After $90 \mathrm{~min}$ \\
\hline SD1 & P1 & $25.9 \pm 0.541$ & $37.4 \pm 1.003$ & $38.6 \pm 0.536$ \\
\hline SD2 & $\mathrm{P} 1$ & $28.6 \pm 0.312$ & $48.7 \pm 0.978$ & $56.3 \pm 0.612$ \\
\hline SD3 & $\mathrm{P} 2$ & $27.8 \pm 1.012$ & $48.9 \pm 0.346$ & $58.5 \pm 0.830$ \\
\hline SD4 & P2 & $25.3 \pm 0.664$ & $34.6 \pm 0.843$ & $41.1 \pm 0.964$ \\
\hline SD5 & $\mathrm{P} 1$ & $15.5 \pm 0.718$ & $30.3 \pm 0.516$ & $42.8 \pm 0.521$ \\
\hline SD6 & P2 & $41.8 \pm 0.992$ & $61.9 \pm 0.982$ & $68.6 \pm 0.843$ \\
\hline SD7 & P2 & $61.2 \pm 0.813$ & $98.5 \pm 0.945$ & $98.8 \pm 1.262$ \\
\hline SD8 & P1 & $28.5 \pm 1.408$ & $42.2 \pm 0.397$ & $43.6 \pm 0.325$ \\
\hline SD9 & P1 & $25.1 \pm 0.119$ & $34.0 \pm 0.863$ & $39.1 \pm 1.463$ \\
\hline SD10 & $\mathrm{P} 2$ & $30.1 \pm 0.761$ & $63.4 \pm 0.214$ & $71.4 \pm 1.332$ \\
\hline SD11 & P2 & $35.4 \pm 0.218$ & $54.9 \pm 0.343$ & $63.6 \pm 0.525$ \\
\hline SD12 & P1 & $28.7 \pm 0.558$ & $39.8 \pm 0.436$ & $41.7 \pm 0.358$ \\
\hline SD13 & P2 & $38.8 \pm 0.592$ & $70.8 \pm 0.951$ & $85.5 \pm 1.175$ \\
\hline SD14 & P1 & $12.6 \pm 1.117$ & $23.5 \pm 1.265$ & $37.3 \pm 0.453$ \\
\hline SD15 & P2 & $32.5 \pm 0.476$ & $52.8 \pm 1.478$ & $63.3 \pm 0.543$ \\
\hline SD16 & P2 & $40.1 \pm 0.845$ & $55.8 \pm 0.659$ & $64.6 \pm 0.866$ \\
\hline SD17 & P1 & $26.0 \pm 0.327$ & $44.4 \pm 1.367$ & $55.5 \pm 0.634$ \\
\hline SD18 & P1 & $19.2 \pm 0.574$ & $34.0 \pm 0.512$ & $39.1 \pm 0.652$ \\
\hline
\end{tabular}

P1 = Soluplus; P2 = Kolliphor TPGS.

drug release of $99.6 \pm 0.935$ in FeSSIF. The extent of increase in the percentage drug release for the drug alone varied from $31.2 \pm 0.425$ in FaSSIF to $47.1 \pm 0.534$ in FeSSIF. The relative increase in the drug release in FaSSIF and FeSSIF media is depicted in Table 3.

3.6. In Vitro Permeation Study. In vitro permeation study by everted gut sac method was done to see the effect of Pgp inhibition characteristic of polymer on the absorption of the drug. The concentration of the drug inside the sac after $2 \mathrm{~h}$ was found to be $451.48 \pm 4.02 \mu \mathrm{g} / \mathrm{ml}$ and when given with Ritonavir, it was found to be $774.51 \pm 2.81 \mu \mathrm{g} / \mathrm{ml}$. This increment in the drug concentration can be owed to the CYP3A4 as well as P-gp inhibiting activity of Ritonavir which enhances the absorption of the drug across the sac. The formulation SD7 showed the highest concentration of $2359.54 \pm 2.95 \mu \mathrm{g} / \mathrm{ml}$ exhibiting a fivefold increase in the drug concentration. Thus, Kolliphor TPGS proved to be a potential $\mathrm{P}$-gp inhibitor in addition to its high solubilizing property.

Apparent Permeability Coefficient. The permeability coefficient $\left(P_{\text {app }}\right)$ was calculated and is represented in Table 4 . The $P_{\text {app }}$ of drug and drug in presence of Ritonavir was found to be $0.828 \times 10^{-6} \mathrm{~cm} / \mathrm{sec}$ and $1.389 \pm 0.08 \times 10^{-6}$, respectively, which is smaller than the permeability coefficient for SD7, that is, $5.899 \pm 0.24 \times 10^{-6} \mathrm{~cm} / \mathrm{sec}$ by about 4 times.

3.7. Pharmacokinetic Studies. The plasma conc. profile of DRV in albino Wistar rats following oral administration of SD7 formulation was compared with the plasma profile obtained following administration of pure drug and drug with RTV in fasting and fed state [Figure 5]. Plasma concentration-time profiles of DRV were evaluated by pharmacokinetic software (PK Functions for Microsoft Excel, Pharsight Corporation, Mountain View, CA). The pharmacokinetic profile clearly showed enhanced bioavailability of the formulations administered during the fed state as compared to the fasted state, thus indicating the significant effect of food on the bioavailability of the drug. The time $\left(T_{\max }\right)$ to reach maximum plasma conc. $\left(C_{\max }\right)$ was found to be the same in all the formulations which was $1 \mathrm{~h}$. The $C_{\max }$ of formulation SD7, that is, $74441.59 \pm 1598.09 \mu \mathrm{g} / \mathrm{ml}$ (Table 5), is 10 times more than $C_{\max }$ value of drug (5874.93 \pm $564.69 \mathrm{ng} / \mathrm{ml}$ ) and almost double $C_{\max }$ value of DRV + RTV $(45161.37 \pm 979.13 \mathrm{ng} / \mathrm{ml})$ in fed state. The high value of AUC (area under the curve) of SD7 in both fasting and fed state confirms greatly enhanced bioavailability of DRV.

Therefore, in vivo study findings demonstrated that Kolliphor TPGS incorporated in solid dispersion formulation of DRV exhibited tremendous potential for improving the solubilization and bioavailability of DRV.

\section{Discussion}

4.1. Selection of Carrier. On the basis of phase solubility studies, Soluplus and Kolliphor TPGS reported the maximum solubility of DRV. However, the solubility of DRV obtained with the carrier Kolliphor TPGS was observed to be slightly higher as compared to that with Soluplus. These results were in accordance with the study reported by Ramesh et al. wherein Kolliphor TPGS showed an enhanced solubility profile as compared to Soluplus in increasing the solubility of Etravirine [21]. 


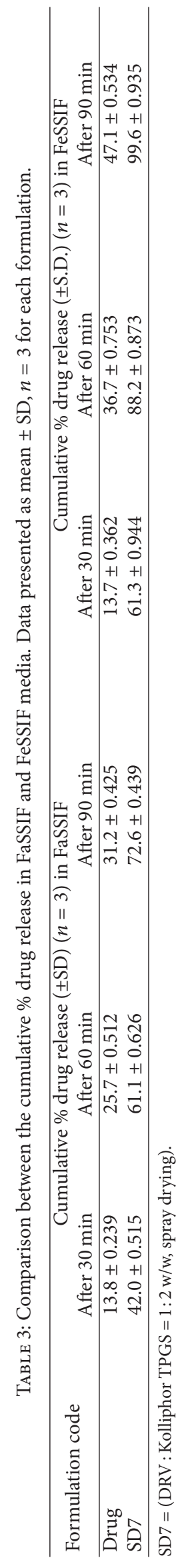




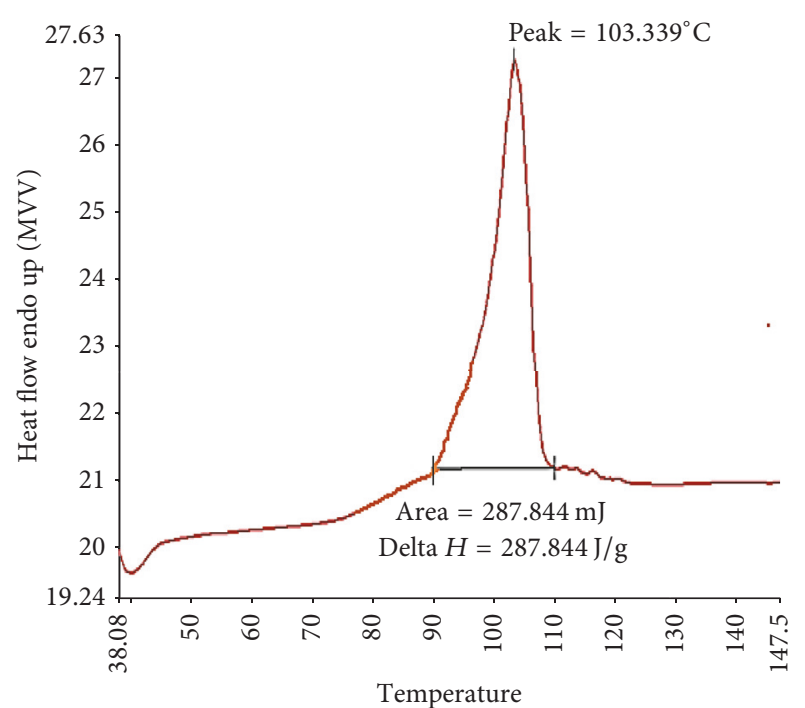

(a)

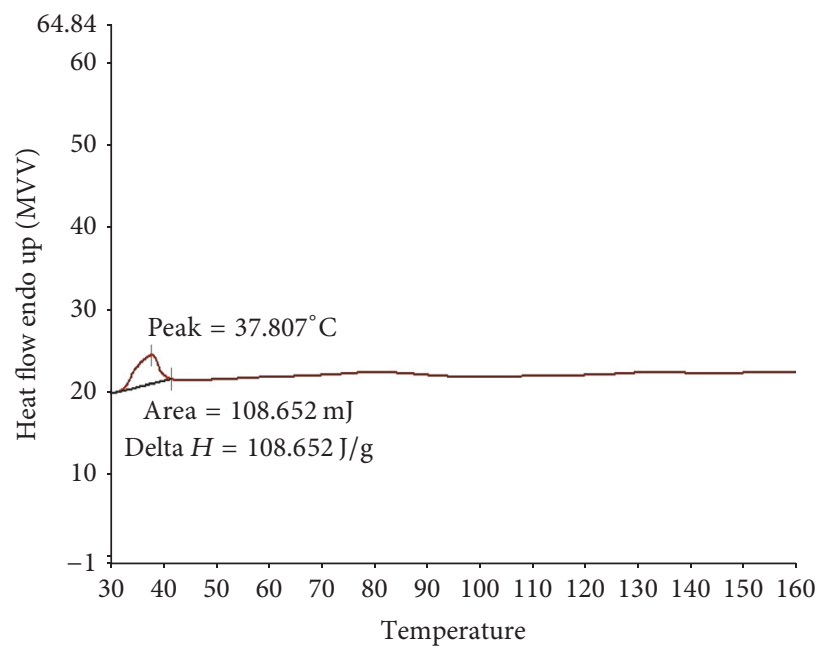

(c)

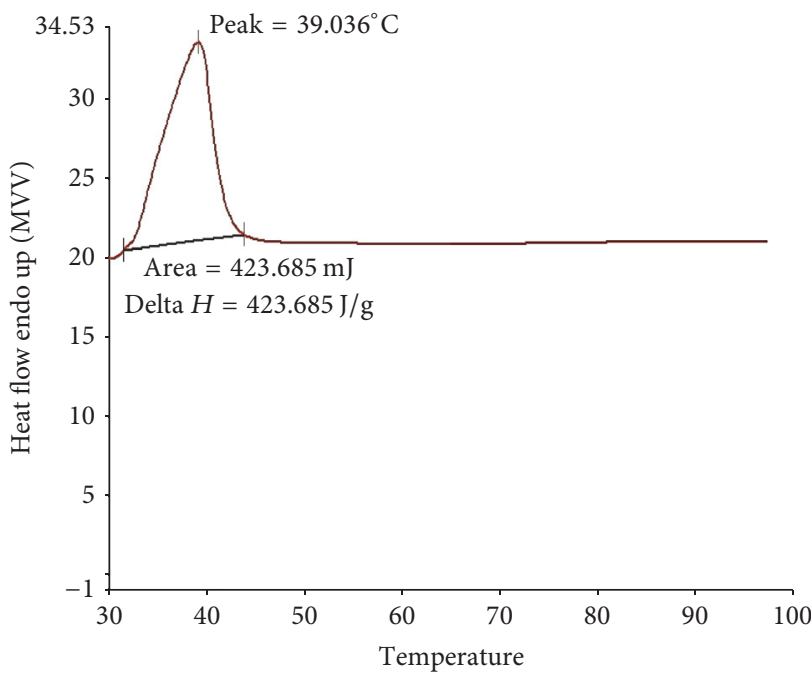

(b)

FIGURE 2: DSC of (a) DRV (b) Kolliphor TPGS and (c) SD7 = SD of DRV with Kolliphor TPGS.

4.2. Custom Design Analysis. The custom design is applied to the 18 formulations of DRV prepared with Soluplus and Kolliphor TPGS as the carrier to obtain the optimized formulation. This design helped in determining model's sensitivity to changes in the factor settings and to identify whether the model is fit for the experiment. The $P$ value and $R^{2}$ value further confirmed the fit of the custom design model for the experiment.

4.3. Optimization of Solid Dispersion. Kolliphor TPGS was selected as the carrier for preparing solid dispersion of DRV attributed to high saturation solubility, drug content, and \% cumulative drug release. Further, in vitro permeation studies and in vivo studies have confirmed the P-gp inhibitory activity of Kolliphor TPGS which has resulted in its remarkable in vivo prospect.
4.3.1. Saturation Solubility Studies. The increase in saturation solubility with increase in polymer concentration might be attributed to the physical properties of solubilizing and emulsifying ability of Kolliphor TPGS. It can also be due to possible complexation of the poorly soluble drug with water soluble carrier. Shin and Kim, 2003, reported a marked enhancement in the solubility from 18.25 to $345.75 \mu \mathrm{g} / \mathrm{mL}$ of 1:2 (w/w) furosemide/TPGS solid dispersion, thus establishing a relevance to our results [22].

4.3.2. Drug Content Determination. The high percent drug content for the SDs containing Kolliphor TPGS indicated uniform distribution of the drug in this hydrophilic carrier without any drug degradation and/or precipitation. Also, low values of standard deviation indicated the reproducibility of the method. Some variations were observed for the dispersions prepared using Soluplus, thus indicating improper 


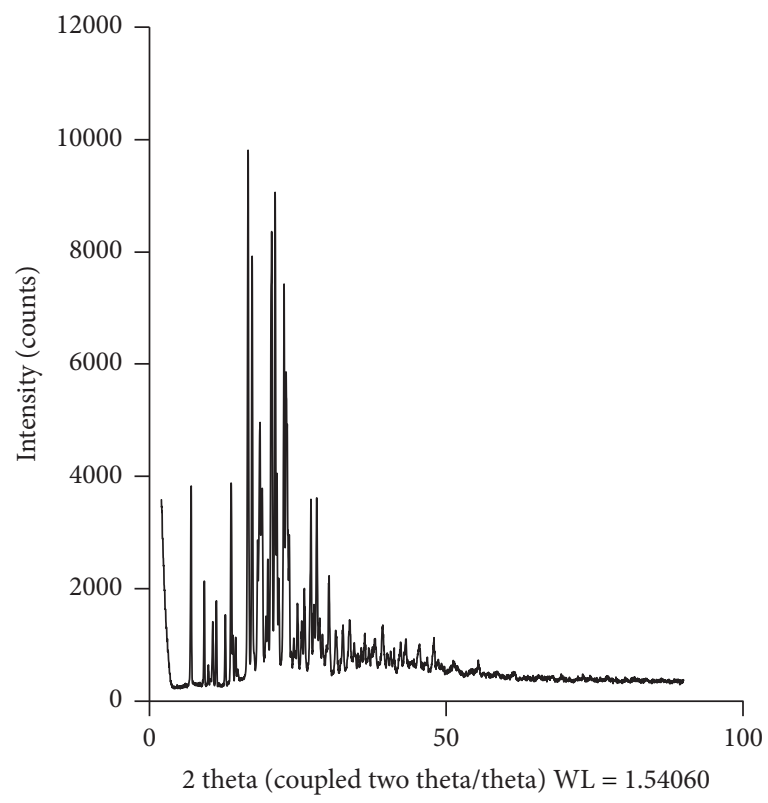

(a)

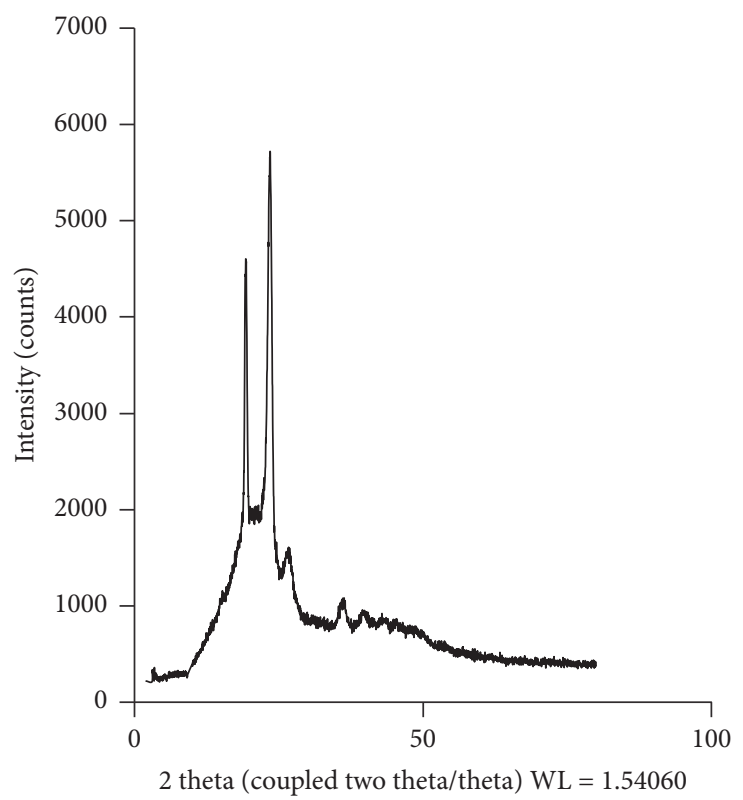

(b)

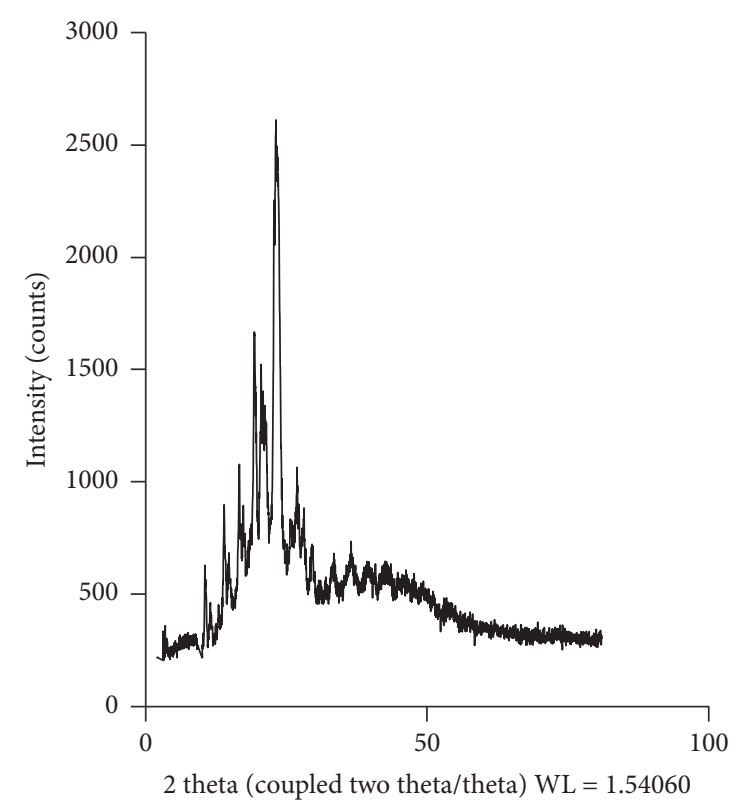

(c)

FIGURE 3: XRD of (a) DRV (b) Kolliphor TPGS and (c) SD7 = SD of DRV with Kolliphor TPGS.

TABle 4: Apparent permeability coefficients $P_{\text {app }}(\mathrm{cm} / \mathrm{s})$. Data presented as mean $\pm \mathrm{SD}, n=3$ for each formulation.

\begin{tabular}{|c|c|c|c|}
\hline Formulation code & $\begin{array}{l}\text { Equation of graph between drug conc. } \\
\text { inside sac }(\mu \mathrm{g} / \mathrm{ml}) \text { and time }(\mathrm{sec})\end{array}$ & $R^{2}$ & $\begin{array}{c}\text { Apparent permeability coefficient } \\
\left(\times 10^{-6} \mathrm{~cm} / \mathrm{sec}\right) \\
\text { mean } \pm \mathrm{SD}\end{array}$ \\
\hline Drug & $0.0375 x+187.53$ & 0.9901 & $0.828 \pm 0.12$ \\
\hline Drug + Ritonavir & $0.0629 x+326.98$ & 0.9959 & $1.389 \pm 0.08$ \\
\hline SD7 & $0.267 x+447.32$ & 0.9995 & $5.899 \pm 0.24$ \\
\hline
\end{tabular}

$\mathrm{SD7}=(\mathrm{DRV}:$ Kolliphor TPGS $=1: 2 \mathrm{w} / \mathrm{w}$, spray drying $)$. 


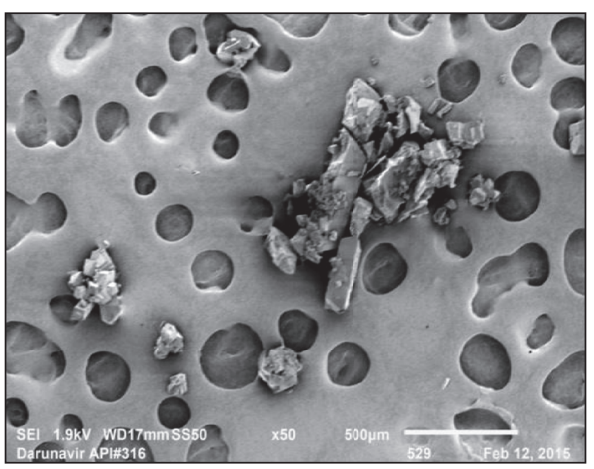

(a)

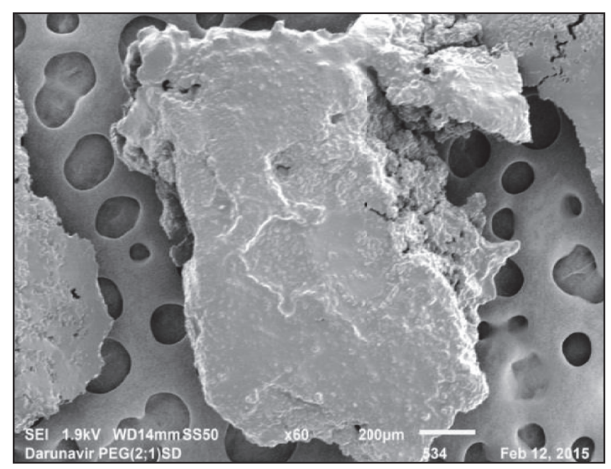

(b)

FIGURE 4: SEM images of (a) DRV and (b) SD7 = SD of DRV Kolliphor TPGS.

TABLE 5: Results for various pharmacokinetic parameters by different formulations in Wistar rats. Data presented as mean \pm SD, $n=3$ for each formulation.

\begin{tabular}{lcccccc}
\hline \multirow{2}{*}{ Groups } & \multirow{2}{*}{ Formulations } & \multicolumn{4}{c}{ Pharmacokinetic parameters } \\
& & $C_{\max }(\mathrm{ng} / \mathrm{ml})$ & $T_{\max }(\mathrm{h})$ & $\mathrm{AUC}_{0-t}(\mathrm{ng} \cdot \mathrm{min} / \mathrm{ml})$ & $\mathrm{AUC}_{0-\alpha}(\mathrm{ng} \cdot \mathrm{min} / \mathrm{ml})$ & $K_{\mathrm{el}}$ \\
\hline \multirow{3}{*}{ Group A (fasted) } & DRV & $2864.28 \pm 365.83$ & 1 & $6945.18 \pm 117.63$ & $7033.51 \pm 119.94$ & $0.211 \pm 0.002$ \\
& $\mathrm{DRV}+\mathrm{RTV}$ & $25115.75 \pm 895.96$ & 1 & $52036.81 \pm 406.93$ & $52182.84 \pm 397.10$ & $0.266 \pm 0.002$ \\
& SD7 & $34820.79 \pm 991.21$ & 1 & $67269.08 \pm 407.25$ & $67500.64 \pm 436.42$ & $0.252 \pm 0.006$ \\
\hline \multirow{3}{*}{ Group B (fed) } & DAR & $5874.93 \pm 564.69$ & 1 & $15451.98 \pm 61.65$ & $15556.26 \pm 59.63$ & $0.228 \pm 0.003$ \\
& DRV + RTV & $45161.37 \pm 979.13$ & 1 & $90985.44 \pm 440.90$ & $91232.40 \pm 465.63$ & $0.264 \pm 0.008$ \\
& SD7 & $74441.59 \pm 1598.09$ & 1 & $172727.90 \pm 919.17$ & $174292.46 \pm 880.76$ & $0.225 \pm 0.001$ \\
\hline
\end{tabular}

$\left(C_{\max }=\right.$ peak plasma concentration; $T_{\max }=$ time taken to reach $C_{\max } ; \mathrm{AUC}_{0-t}=$ area under curve from $0-t ; \mathrm{AUC}_{0-\alpha}=$ area under curve from $0-\alpha ; K_{\mathrm{el}}=$ elimination rate constant).

distribution of the drug within the carrier. Similar results were obtained by Barea and coworkers who reported the drug content of thalidomide to be $117.2 \pm 8.1 \%$ in the thalidomide: Kolliphor TPGS (1:4) solid dispersion owing to the uniform distribution of drug within the hydrophilic carrier [23].

4.3.3. In Vitro Dissolution Study. On the basis of DoE study, it was seen in Figure 1 that all input variables exhibited a marked effect on the response variables. The response variables generated for the runs of the custom design were analyzed to get the optimized formulation along with the method of preparation. For SDs prepared using Kolliphor TPGS, polymer:drug ratio showed a remarkable increase in \% drug release when spray drying method was used for formulation preparation. The reason for the high \% of drug release with Kolliphor TPGS can be attributed to the improvement of wetting of drug particles due to surfaceactive property of polymer and localized solubilization by the hydrophilic nature of the polymer. Savjani et al. similarly optimized solid dispersion of itraconazole based on different polymers using DoE [24].

4.4. Characterization of Optimized Solid Dispersion. The optimized formulation, that is, SD7, on characterization by DSC showed disappearance of sharp melting peak of drug indicating a reduced degree of crystallinity and that the drug is either solubilized due to the presence of used excipients or present in an amorphous form. Fule and Amin observed similar results with solid dispersion of Lafutidine (LAFT) prepared by hot melt processing approach. The disappearance of peak of LAFT confirmed its conversion to its amorphous form [25].

Absence of peak characteristic of DRV and shift in the peaks of Kolliphor in FTIR spectra indicated presence of some interaction between drug and polymer. Bond formation between the drug and polymer might have occurred between drug and polymer. Similarly, Li et al. elucidated the reason behind disappearance of peaks of Curcumin in FTIR study to be as a result of interaction due to phenolic, carbonyl, and H-bond between Curcumin and Eudragit ${ }^{\circledR}$ E PO [26].

XRD revealed there might be transformation of crystalline DRV into amorphous form during the spray drying process as there was disappearance of peaks characteristic to DRV. The reason for this could be that spray drying is an energy intensive process where solution passes from the state of relative unsaturation to supersaturation in a fraction of seconds. Further, rapid evaporation of solvent from the supersaturated atomized droplets of the solution seemingly interferes with the crystal building process leading to amorphization of the drug. These results were in accordance with the results of Shamma and Basha who found out that the diffractogram of the solid dispersion of Carvedilol (CAR) showed complete absence of the distinctive peaks of CAR. The typical diffuse pattern obtained with the solid dispersion 


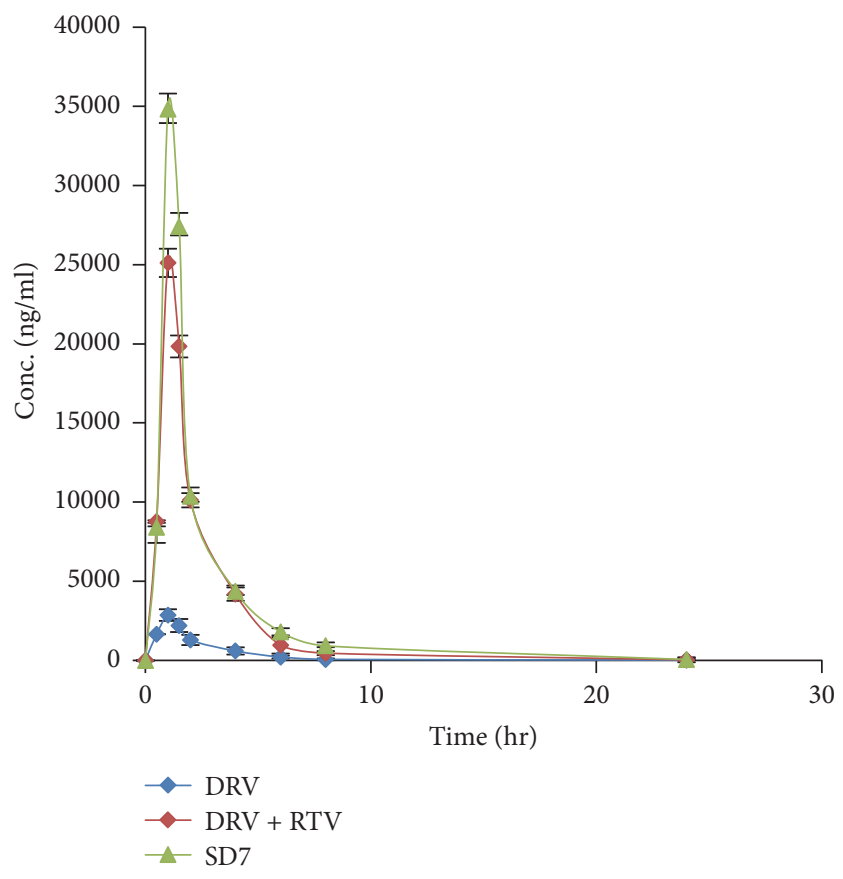

(a)

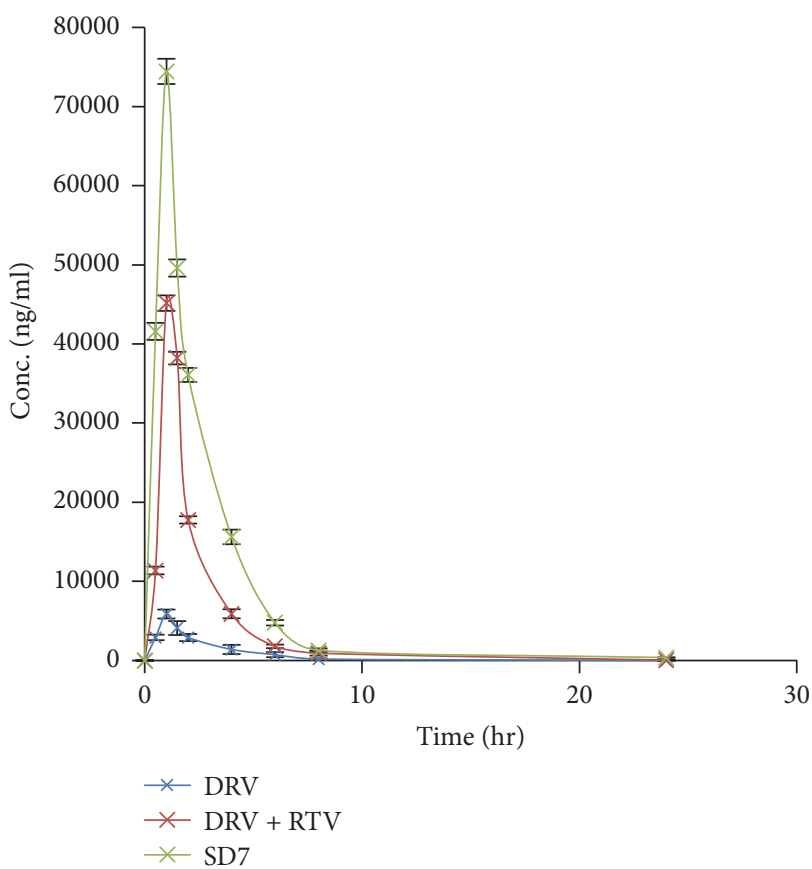

(b)

FIGURE 5: Plasma concentration profile of DRV following oral administration of different formulations in (a) fasted state and (b) fed state. Data presented as mean $\pm \mathrm{SD}, n=3$ for each formulation.

indicated the disruption of crystalline nature of CAR and changing into an entirely amorphous state [27].

SEM microphotographs revealed the presence of drug particles dispersed in the polymer matrix which was confirmed by the smooth texture exhibited in SD. There is no evidence of drug crystals, which confirms the previous findings based on XRD patterns. Similar results were obtained with the SEM results of solid dispersion of CoQ10 which confirmed the existence of CoQ10 in an amorphous form or very fine crystalline form [28].

4.5. Dissolution Studies in FaSSIF and FeSSIF Media. Increased release of DRV in FeSSIF confirms the higher concentration gradient of drug at the absorption site which will ultimately lead to an increased absorption. Furthermore, FeSSIF media facilitate this absorption by enhancing the solubilization of drug. So it was concluded that the formulation will be better absorbed when given with food. The study is in concordant with the study elucidated by Sinha and coworkers who demonstrated a better \% drug release of solid dispersion of Ritonavir with Gelucire in FeSSIF media than FaSSIF indicating the effect of food on drug absorption [17]. Sekar et al. have also confirmed the increase in the absorption of DRV in the presence of food in a study done on healthy human volunteers [5].

4.6. In Vitro Permeation Study. The reason for the considerably lower value of $P_{\text {app }}$ of DRV is owed to the presence of P-gp efflux transporters on the brush border membrane of intestinal epithelium which is responsible for attenuating the permeability of DRV. It also increases its level of exposure to cellular and luminal enzymes by effluxing the drug into the intestinal lumen or blood capillaries, thus metabolizing the drug [29]. The fourfold higher $P_{\text {app }}$ value obtained with SD7 demonstrated that P-gp inhibition by Kolliphor TPGS could be considered as the most likely mechanism for enhancement of DRV absorption which allowed drug to permeate across the sac more easily and assisted in inhibiting the P-gp efflux of the drug. Thus, Kolliphor TPGS was proved to be a good choice as a polymer for enhancing the absorption of DRV. The potential of Kolliphor TPGS as a P-gp inhibitor was in accordance with that obtained by Varma and Panchagnula. Intrinsic permeability of paclitaxel in the presence of $1 \mathrm{mg} / \mathrm{ml}$ TPGS was found to increase from $0.08 \times 10^{-4}$ to $0.22 \times$ $10^{-4} \mathrm{~cm} / \mathrm{s}$. Thus, they demonstrated significant improvement in intestinal permeability of paclitaxel using Kolliphor TPGS [30].

4.7. Pharmacokinetic Studies. The study suggested that improved bioavailability in case of the SD of DRV in both fasted and fed states can be attributed primarily to the solubilizing activity and P-gp inhibiting action of Kolliphor TPGS incorporated in the dispersion. The carrier molecules fluidize the plasma membrane by inserting themselves between tails of the lipid bilayer, interacting with the bilayer's polar heads and thus modifying the hydrogen/ionic bond forces which may add onto their inhibitory action [31]. Also, the presence of food and moreover the possible size reduction might have occurred during the preparation which lead to easy availability of the drug and immediate drug release in the systemic circulation [32]. 
The in vivo data clearly indicated that Kolliphor TPGS can serve as a potential carrier for improving the solubility and bioavailability not only of orally administered Darunavir but also of other BCS class II-IV drugs which are P-gp substrates. The result was in concordance with that of the results obtained by Kim et al. wherein the effect of simultaneous administration of curcumin with P-gp substrate drug Saquinavir was studied. Curcumin owing to its P-gp inhibiting activity was found to increase the oral exposure of Saquinavir in rats by 2.7 -fold [32].

\section{Conclusion}

Substantial solubility enhancement as well as P-gp inhibition was achieved for the drug DRV by formulating it into solid dispersion using Kolliphor TPGS as a carrier possessing Pgp inhibiting activity. The industrially scalable method, that is, spray drying, was optimized for the preparation of $\mathrm{SD}$ using DoE. Findings of DSC, XRD, FTIR, NMR, and SEM confirmed that DRV was present in an amorphous state in the spray dried SD at a drug: polymer ratio of $1: 2(\mathrm{w} / \mathrm{w})$. The saturated solubility of DRV was markedly enhanced. The dissolution performed in biorelevant media exhibited maximum dissolution with FeSSIF media than in FaSSIF which confirmed food-related absorption of drugs. The Pgp inhibiting activity of the Kolliphor TPGS polymer was confirmed by both in vitro permeation studies and in vivo studies. The effect of food on absorption of drugs was also confirmed by conducting the pharmacokinetic studies in animals in both fasted and fed state. Thus, solid dispersions prepared using Kolliphor TPGS as a polymeric carrier and P-gp inhibitor together with spray drying as the preparation method represent a promising approach for enhancing the solubility, reducing the P-gp efflux, and finally improving the therapeutic efficacy of DRV.

\section{Conflicts of Interest}

The authors declare that there are no conflicts of interest regarding the publication of this article.

\section{Acknowledgments}

The authors are grateful to All India Council of Technical Education (AICTE), New Delhi, India, for providing financial assistance for the present study.

\section{References}

[1] S. Kotta, A. W. Khan, S. H. Ansari, R. K. Sharma, and J. Ali, "Anti HIV nanoemulsion formulation: optimization and in vitro-in vivo evaluation," International Journal of Pharmaceutics, vol. 462, no. 1-2, pp. 129-134, 2014.

[2] M. Lamorde, P. Byakika-Kibwika, V. Okaba-Kayom et al., "Nevirapine pharmacokinetics when initiated at $200 \mathrm{mg}$ or 400 mg daily in HIV-1 and tuberculosis co-infected Ugandan adults on rifampicin," Journal of Antimicrobial Chemotherapy, vol. 66, no. 1, Article ID dkq411, pp. 180-183, 2011.
[3] M. H. A. Biswas, "AIDS epidemic worldwide and the millennium development strategies: a light for lives," HIV \& AIDS Review, vol. 11, no. 4, pp. 87-94, 2012.

[4] L. Griffin, P. Annaert, and K. L. R. Brouwer, "Influence of drug transport proteins on the pharmacokinetics and drug interactions of HIV protease inhibitors," Journal of Pharmaceutical Sciences, vol. 100, no. 9, pp. 3636-3654, 2011.

[5] V. Sekar, D. Kestens, S. Spinosa-Guzman et al., "The effect of different meal types on the pharmacokinetics of darunavir (TMC114)/ritonavir in HIV-negative healthy volunteers," Clinical Pharmacology and Therapeutics, vol. 47, no. 4, pp. 479-484, 2007.

[6] D. Back, V. Sekar, and R. M. W. Hoetelmans, "Darunavir: Pharmacokinetics and drug interactions," Antiviral Therapy, vol. 13, no. 1, pp. 1-13, 2008.

[7] H. Fujimoto, M. Higuchi, H. Watanabe et al., "P-glycoprotein mediates efflux transport of Darunavir in human intestinal Caco-2 and ABCB1 gene-transfected renal LLC-PK1 cell lines," Biological \& Pharmaceutical Bulletin, vol. 32, no. 9, pp. 15881593, 2009.

[8] A. Kogawa and H. R. N. Salgadol, "Characteristics, complexation and analytical methods of darunavir," British Journal of Pharmaceutical Research, vol. 4, no. 11, pp. 1276-1286, 2014.

[9] J. Kulkosky and S. Bray, "HAART-persistent HIV-1 latent reservoirs: their origin, mechanisms of stability and potential strategies for eradication," Current HIV Research, vol. 4, no. 2, pp. 199-208, 2006.

[10] C. Leuner and J. Dressman, "Improving drug solubility for oral delivery using solid dispersions," European Journal of Pharmaceutics and Biopharmaceutics, vol. 50, no. 1, pp. 47-60, 2000.

[11] M. Vasanthavada, W.-Q. Tong, Y. Joshi, and M. S. Kislalioglu, "Phase behavior of amorphous molecular dispersions I: determination of the degree and mechanism of solid solubility," Pharmaceutical Research, vol. 21, no. 9, pp. 1598-1606, 2004.

[12] K. M. Raghava and P. K. Lakshmi, "Overview of P-glycoprotein inhibitors: a rational outlook," Brazilian Journal of Pharmaceutical Sciences, vol. 48, no. 3, pp. 353-367, 2012.

[13] M. V. S. Varma, Y. Ashokraj, C. S. Dey, and R. Panchagnula, "P-glycoprotein inhibitors and their screening: a perspective from bioavailability enhancement," Pharmacological Research, vol. 48, no. 4, pp. 347-359, 2003.

[14] A. C. Kogawa, A. Zoppi, M. A. Quevedo, M. R. Longhi, and H. R. N. Salgadol, "Complexation between darunavir ethanolate and B-cyclodextrin Experimental and theoretical studies," World Journal of Pharmacy and Pharmaceutical Sciences, vol. 3, no. 6, pp. 298-309, 2014.

[15] M. Thommes, L. Baert, G. van 't Klooster et al., "Improved bioavailability of darunavir by use of $\kappa$-carrageenan versus microcrystalline cellulose as pelletisation aid," European Journal of Pharmaceutics and Biopharmaceutics, vol. 72, no. 3, pp. 614620, 2009.

[16] L. D. S. Alves, M. F. De La Roca Soares, C. T. De Albuquerque et al., "Solid dispersion of efavirenz in PVP K-30 by conventional solvent and kneading methods," Carbohydrate Polymers, vol. 104, no. 1, pp. 166-174, 2014.

[17] S. Sinha, M. Ali, S. Baboota, A. Ahuja, A. Kumar, and J. Ali, "Solid dispersion as an approach for bioavailability enhancement of poorly water-soluble drug ritonavir," AAPS PharmSciTech, vol. 11, no. 2, pp. 518-527, 2010.

[18] B. N. Patel, B. N. Suhagia, and C. N. Patel, "RP-HPLC method development and validation for estimation of darunavir 
ethanolate in tablet dosage form," International Journal of Pharmacy and Pharmaceutical Sciences, vol. 4, no. 3, pp. 270273, 2012.

[19] S. Gurunath, B. K. Nanjwade, and P. A. Patila, "Enhanced solubility and intestinal absorption of candesartan cilexetil solid dispersions using everted rat intestinal sacs," Saudi Pharmaceutical Journal, vol. 22, no. 3, pp. 246-257, 2014.

[20] M. Takahashi, Y. Kudaka, N. Okumura, A. Hirano, K. Banno, and T. Kaneda, "The validation of plasma darunavir concentrations determined by the HPLC method for protease inhibitors," Biological \& Pharmaceutical Bulletin, vol. 30, no. 10, pp. 19471949, 2007.

[21] K. Ramesh, B. C. Shekar, P. Khadgapathi, D. V. R. N. Bhikshapathi, and N. Gourav, "Enhancement of Solubility and Bioavailability of Etravirine Solid Dispersions by Solvent Evaporation Technique with Novel Carriers," IOSR Journal of Pharmacy and Biological Sciences, vol. 10, no. 4, pp. 30-41, 2015.

[22] S.-C. Shin and J. Kim, "Physicochemical characterization of solid dispersion of furosemide with TPGS," International Journal of Pharmaceutics, vol. 251, no. 1-2, pp. 79-84, 2003.

[23] S. A. Barea, C. B. Mattos, A. C. C. Cruz et al., "Solid dispersions enhance solubility, dissolution, and permeability of thalidomide," Drug Development and Industrial Pharmacy, vol. 43, no. 3, pp. 511-518, 2017.

[24] K. Savjani, A. Gajjar, and J. Savjani, "Design and optimization of itraconazole tablet employing solid dispersion approach," Asian Journal of Pharmaceutics, vol. 11, no. 1, pp. S192-S207, 2017.

[25] R. Fule and P. Amin, "Development and evaluation of lafutidine solid dispersion via hot melt extrusion: investigating drugpolymer miscibility with advanced characterisation," Asian Journal of Pharmaceutical Sciences, vol. 9, no. 2, pp. 92-106, 2014.

[26] J. Li, I. W. Lee, G. H. Shin, X. Chen, and H. J. Park, "CurcuminEudragit ${ }^{\circledR}$ E PO solid dispersion: A simple and potent method to solve the problems of curcumin," European Journal of Pharmaceutics and Biopharmaceutics, vol. 94, pp. 322-332, 2015.

[27] R. N. Shamma and M. Basha, "Soluplus ${ }^{\circledR}$ : A novel polymeric solubilizer for optimization of Carvedilol solid dispersions: Formulation design and effect of method of preparation," Powder Technology, vol. 237, pp. 406-414, 2013.

[28] P. R. Nepal, H.-K. Han, and H.-K. Choi, "Enhancement of solubility and dissolution of Coenzyme Q10 using solid dispersion formulation," International Journal of Pharmaceutics, vol. 383, no. 1-2, pp. 147-153, 2010.

[29] S. Bhise, D. Mathure, M. V. K. Patil, and R. D. Patankar, "Solubility enhancement of antihypertensive agent by solid dispersion technique," International Journal of Pharmacy \& Life Sciences, vol. 2, no. 8, pp. 970-975, 2011.

[30] M. V. S. Varma and R. Panchagnula, "Enhanced oral paclitaxel absorption with vitamin E-TPGS: effect on solubility and permeability in vitro, in situ and in vivo," European Journal of Pharmaceutical Sciences, vol. 25, no. 4-5, pp. 445-453, 2005.

[31] Y.-L. Lo, "Relationships between the hydrophilic-lipophilic balance values of pharmaceutical excipients and their multidrug resistance modulating effect in Caco-2 cells and rat intestines," Journal of Controlled Release, vol. 90, no. 1, pp. 37-48, 2003.

[32] S.-A. Kim, S.-W. Kim, H.-K. Choi, and H.-K. Han, "Enhanced systemic exposure of saquinavir via the concomitant use of curcumin-loaded solid dispersion in rats," European Journal of Pharmaceutical Sciences, vol. 49, no. 5, pp. 800-804, 2013. 

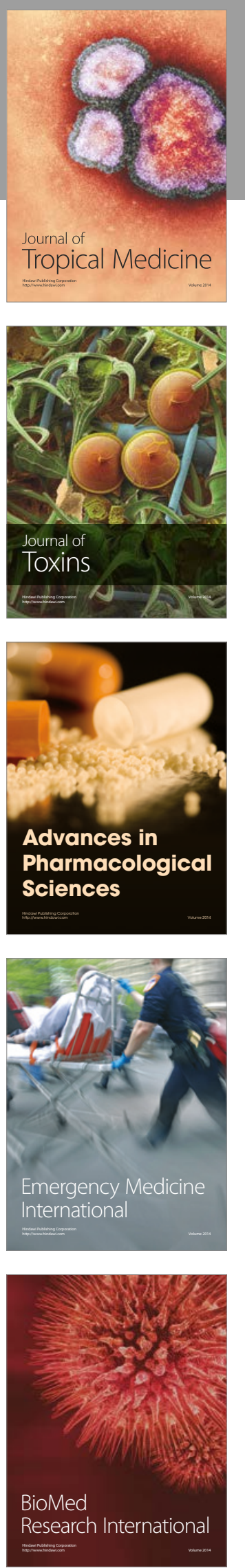
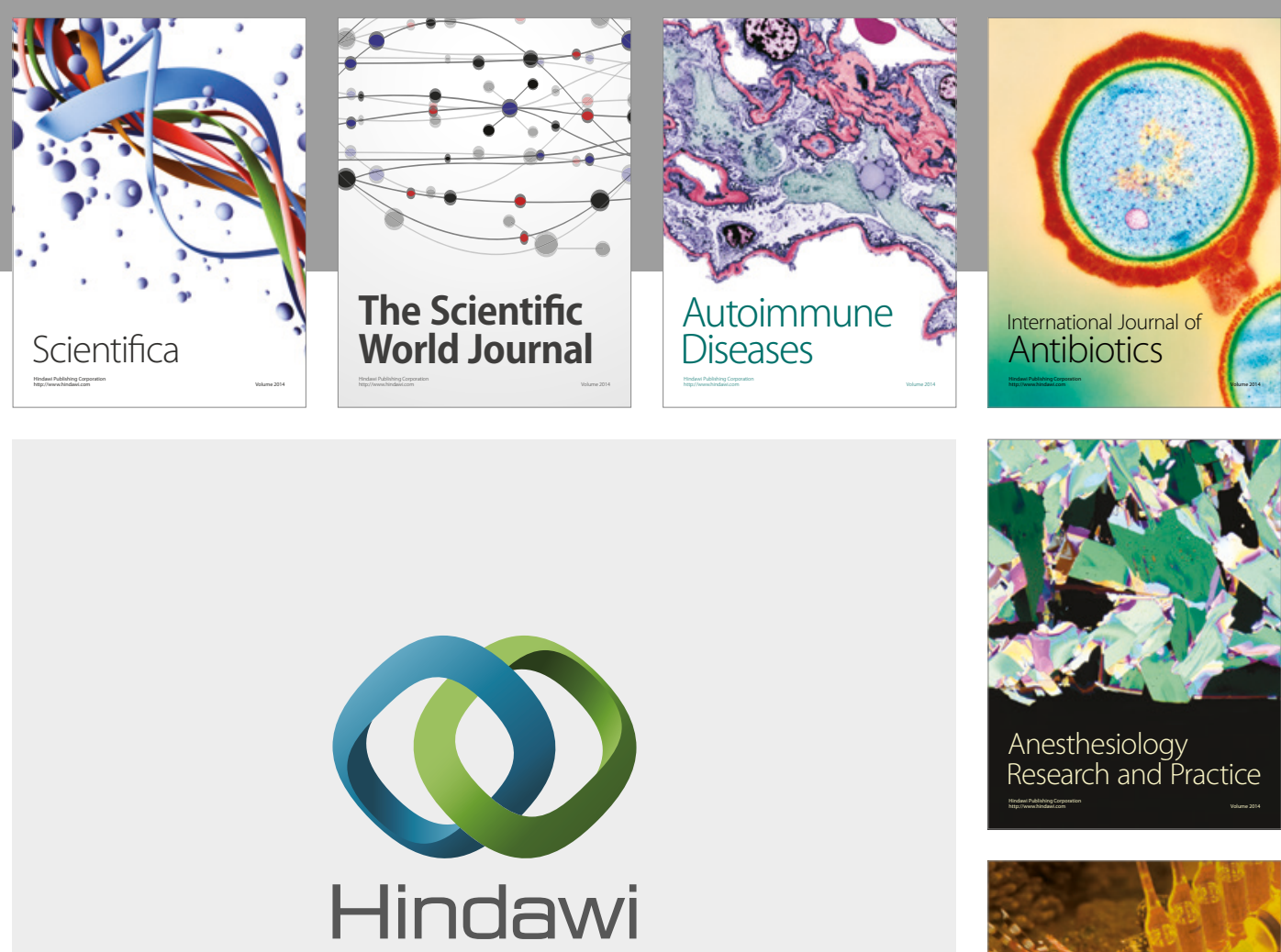

Submit your manuscripts at

https://www.hindawi.com
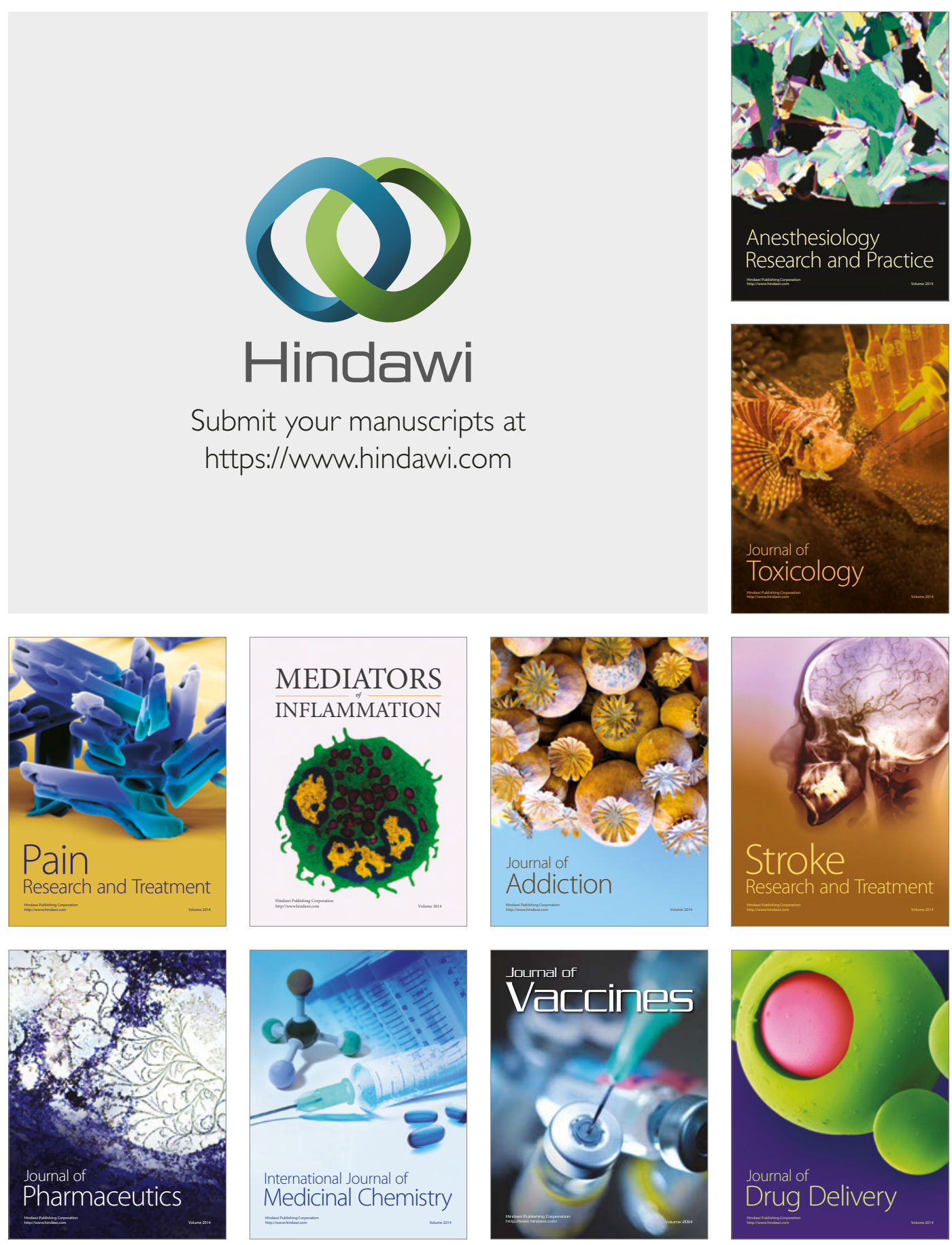